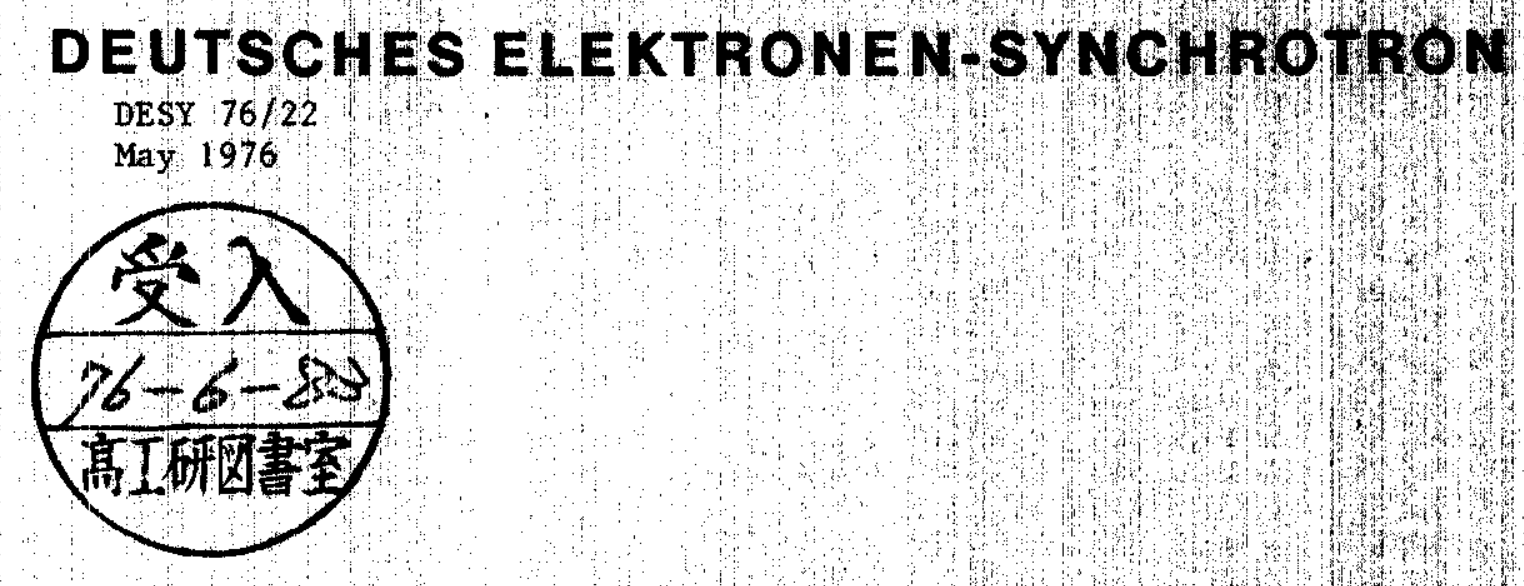

Collision Theory for Mass 1 ess Bdsons

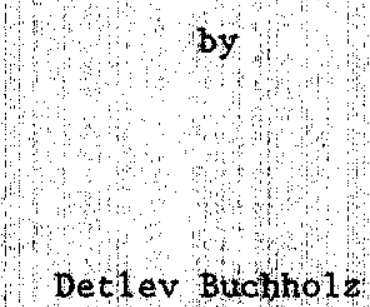

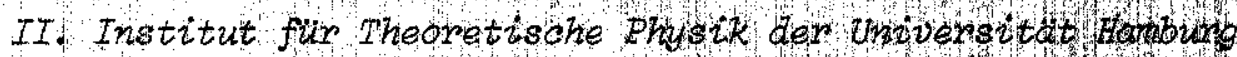

2 HAMBURG 52 NOTKSTIECI
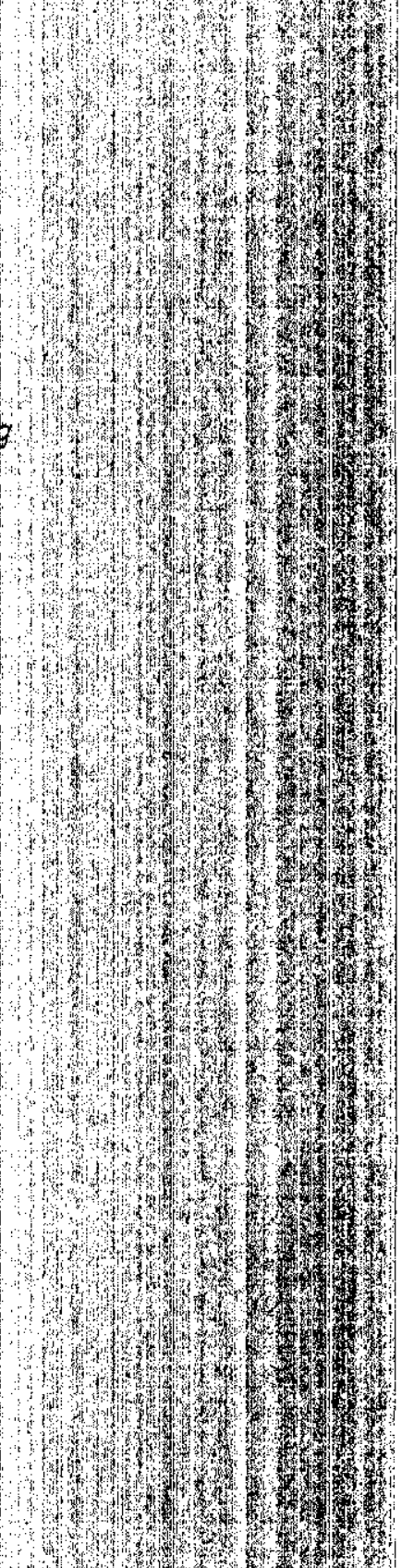
(3)

To besure that your preprints are promptly included in the HIGH ENERGY PHYSICS INDEX,

send them to the following address (if possible by air mail) :

DESY

Bibliothek

2 Hamburg 52

Notkestieg 1

Germany 


\section{COLLISION THEORY FOR MASSLESS BOSONS}

by

Detlev Buchholz

II. Institut für Theoretische Physik der Universität Hamburg

Abstract

We present a complete solution of the collision problem for massless Bosons in four space-time dimensions. 


\section{Introduction}

We continue here our discussion of the collision problem for massless particles in the setting of local, relativistic quantum theory. In two previous papers we developed a collision theory for massless Fermions [1] and for waves in two space-time dimensions [2]. It is the aim of the present article to extend this analysis to models including also massless Bosons.

As soon as massless Bosons take part in collisions one is faced with all kinds of infrared problems. The most spectacular one is the disintegration of charged massive particles into infraparticles [3]. A famous example of this phenomenon can be met in quantum electrodynamics where it is indicated by perturbation theory that the electron does not have a precise mass due to the Coulomb field which it carries along. The massless particles however manifest themselves as real particles with a precise mass in most of the models of physical interest: they appear either as a consequence of a gauge symmetry of the second kind or they result from a spontaneously broken ordinary symmetry via the Goldstone mechanism [4]. It is therefore no essential loss of generality if we restrict our attention to models in which at least the massless particles can be sharply defined as proper eigenstates of the mass operator.

Another difficulty in the presence of massless particles is connected with the construction of charged states from the vacuum. It is well known that locality of the charge carrying fields is in general not compatible with positivity of the metric in the state space. In quantum electrodynamics for example, one has either to abandon locality of the Fermi fields (as in the Coulomb-gauge) or one looses positivity of the metric (as in the Gupta-Bleuler gauge) [5]. For this reason gauge theories like quantum electrodynamics do not fit completely into the framework which is used in this paper. However we want to emphasize that our arguments apply to the vacuum representation of the gauge invariant quantities in these models.

As in our previous investigations we shall use the Huyghens principle and locality in order to establish the existence of asymptotic fields corresponding to the massless Bosons. However, in contrast to the models treated so far, the present construction is burdened with many technicalities owing to the fact that the asymptotic Bose fields are unbounded operators. It will be one of the main tasks of our analysis to extract informations from the basic postulates about the structure of their domain of definition. 
In order to solve these problems we need some estimates for vacuum expectation values of local operators at large spacelike distances. Uniform estimates for arbitrary configurations of the operators (similar to the massive case) are too weak and of no use here. However, we shall see that suitable spherical means of the vacuum expectation values have clustering properties which are sufficient for our purposes. These estimates, which are given in the Appendix, will enable us to construct collision states of massless Bosons with the familiar Fock structure. We shall then see that the (real) asymptotic fields are essentially selfadjoint on their natural domain of definition which is given by the Huyghens principle and locality. This somewhat technical result will simplify our proof that the asymptotic field operators have all the properties of a free, massless field. It will furthermore enable us to construct the asymptotic field algebras and to establish their local, covariant net structure.

An analysis of the physically relevant representations of the asymptotic field algebras would be the natural next step in the discussion of the collision problem for massless particles. If infinitely many massless particles can be produced in collisions one expects that there appear besides the Fock representation (induced by the vacuum) other representations in which a particle number operator cannot be defined. It would be desirable to gain some knowledge about the structure of these infrared representations within the general framework of local field theory. Unfortunately, our investigations of these questions are not yet complete. We mention as an interesting partial result that the representations of the asymptotic field algebras, which are induced by vectors in the physical state space, have the local Fock property. This means that the restrictions of any physical state of the asymptotic field algebras attached to finite spacetime regions (and even to certain unbounded regions) can be interpreted as incoming and outgoing configurations of massless particles.

As in Ref.[1] we express the basic field theoretical structures in terms of a field algebra $\mathcal{F}$ of bounded operators acting irreducibly on a separable Hilbert space $\mathcal{H}$ of physical states. $\mathcal{F}$ is generated by a net $\boldsymbol{O} \rightarrow \mathfrak{F}(\boldsymbol{O})$ of local algebras attached to the open, bounded regions $G \subset \mathbb{R}^{4}$. We assume that a11 local operators commute at spacelike distances:

$$
\mathfrak{F}\left(O_{1}\right)=\mathcal{F}\left(O_{2}\right)^{\prime} \text { for } O_{1} \subset O_{2}^{\prime} \text {. }
$$


Models including also Fermi operators would require only an additional expense of notation). Furthermore we assume that there exists a continuous unitary representation $L \rightarrow U(L)$ of the Poincaré group $\mathcal{P}$ in $\mathscr{H}$ which induces automorphisms of the local net:

$$
\alpha_{L}(\mathcal{F}(0))=U(L) \tilde{F}(G) U(L)^{-1}=\mathcal{F}(L \sigma), L \in P .
$$

The spectrum of the generators of the translations $\left(x_{0}, \underline{x}\right)=x \rightarrow U(x)$ is contained in the closed forward lightcone. There exists an (up to a phase) unique unit vector $\Omega$, the vacuum, which is invariant under the action of

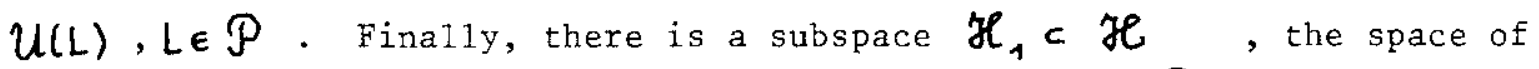
massless one-particle states, on which the $U(L), L \in P$ act like a representation of the Poincaré group $\mathcal{P}$ with mass $m=0$.

\section{The Asymptotic Fields}

This section is devoted to the construction of asymptotic field operators corresponding to the massless particles and to a preliminary analysis of their domain of definition. As in Ref.[1] we define the asymptotic fields as adiabatic limits of operators $A \in \mathcal{F}$. Actually we shall take not any $A \in \mathcal{F}$ but only local operators for which the operator valued functions $x \rightarrow A(x)=U(x) A U(x)^{-1}$ are arbitrarily often differentiable in the uniform topology. These operators constitute $a^{*}$-algebra $\mathfrak{F}_{0}$, which is invariant under Poincaré transformations and weakly dense in $\mathfrak{F}$. So $\mathfrak{F}_{0}$ contains all essential informations about $\mathfrak{F}$.

Now let $A$ be any operator from $\mathfrak{F}_{0}$. We define for each $t \in \mathbb{R}$ a spherical mean of $A$,

$$
A_{t}=-2 t \cdot \int d \omega \partial_{0} A(t, t \underline{e})
$$

Here $d \omega=d \omega(\underline{e})$ is the normialized, invariant measure on the unit sphere $S^{2}$ in $\mathbb{R}^{3}$, e a unit vector which runs over the sphere and $\partial_{0}$ denotes differentiation with respect to the time component of the translations; the integral is defined as Bochner-integral. If one applies $A_{t}$ to the vacuum one gets

$$
A_{t} \Omega=|\underline{P}|^{-1} \cdot\left(e^{i t(H-|\underline{P}|)}-e^{i t(H+|\underline{P}|)}\right) H A \Omega
$$


where $H$ is the Hamiltonian and $\underline{P}$ the momentum operator. The right hand side of this equation is well defined because the operator in the bracket maps the vectors in $\mathbb{X}$ into the domain of $|\underline{P}|^{-1}$. However, in the course of our analysis it will be necessary to interchange the order of the bracket and $|\underline{P}|^{-1}$ and the question arises whether the vector $H A \Omega$ is still in the domain of $|\underline{P}|^{-1}$. One could always achieve this by smearing $A$ with a suitable testfunction. Yet such.a smearing is not even necessary. Using similar arguments as Araki, Hepp and Ruelle in Ref.[6] one can show that for arbitrary local operators $A \in \widetilde{F}_{0}$.

$$
|(H A \Omega, u(\underline{x}) H A \Omega)| \leq c \cdot(1+|\underline{x}|)^{-3},
$$

and from this estimate it follows after Fourier transformation that $H A \Omega$ is an element of $D\left(|\underline{P}|^{-1}\right)$.

In the next step we integrate $t \rightarrow A_{t}$ with a function $h_{\boldsymbol{r}}$ which is defined by

$$
h_{\Gamma}(t)=(\ln |T|)^{-1} \cdot h\left((\ln |T|)^{-1} \cdot(t-T)\right),|T|>1
$$

Here $h$ is an arbitrary real, smooth function with compact support which is normalized according to $\int d t h(t)=1$. Thus $h_{r}$ has support in an interval around $T$ of a length proportional to $\ln |T|$. (It is of no relevance that we have taken the logarithm in the definition of $h_{\mathrm{T}}$. Any other slowly increasing function would do the same job). We set

$$
A_{T}=\int d t h_{T}(t) A_{t},|T|>1
$$

where the integral is defined as Bochner integral. It follows then from relation (4) that

$$
A_{T} \Omega=\int d t h_{T}(t)\left(e^{i t(H-|\underline{P}|)}-e^{i t(H+|\underline{P}|)}\right) \cdot|\underline{\underline{P}}|^{-1} H A \Omega .
$$

So the mean ergodic theorem [7] or the explicit calculations in Ref.[1] can be used to establish the existence of the strong limits

$$
s-\lim _{T \rightarrow \pm \infty} A_{\uparrow} \Omega=P_{1} \cdot\left|\underline{P_{1}}\right|^{-1} H A \Omega=P_{1} A \Omega
$$


where $P_{1}$ is the projection onto the space $\mathscr{Z}_{1}$ of massless one-particle states. Now if $A$ is localized in some bounded region $\boldsymbol{O}, A \in \mathcal{F}(6)$, it follows from relations (3) and (6) that $A_{\boldsymbol{r}}$ is localized in a region which is for sufficiently large $T$ spacelike separated from any given bounded region $\boldsymbol{U}_{1}$ in the future tangent $\mathcal{O}_{+}$of $\mathcal{O}^{.1)}$ So owing to locality one gets for all $F \in \mathcal{F}\left(\sigma_{+}\right)=\bigcup_{\sigma_{1} \subset \sigma_{+}} \mathcal{F}\left(\sigma_{1}\right)$

$$
S-\lim _{T \rightarrow \infty} A_{T} F \Omega=S-\lim _{T \rightarrow \infty} F A_{T} \Omega=F P_{1} A \Omega
$$

and this relation defines a linear operator $A^{\text {out }}$ on the dense set of vector $\left\{F \Omega: F \in \mathcal{F}\left(G_{+}\right)\right\}$. In our first lemma we list some properties of this operator.

\section{Lemma 1:}

Let $A \in \mathcal{F}_{0}$ be localized in some bounded region $\mathcal{O} \subset \mathbb{R}^{4}, A \in \mathcal{F}(\mathcal{O})$.

a) Then the operator $A^{\text {out }}$, which is defined on the dense set of vectors $\{F \Omega: F \in \mathcal{F}(6)\}$ by

$$
A^{\text {out }} F \Omega=s-\lim _{T \rightarrow \infty} A_{T} F \Omega=F P_{1} A \Omega
$$

is closable. We denote the least closed extension of this operator also by $\mathrm{A}^{\text {out }}$ and its domain by $\mathrm{D}\left(\mathrm{A}^{\text {out }}\right)$.

b) $\left(A^{* \text { out }}\right)^{*} \supset A^{\text {out }}$ : if in particular $A^{*}=A$ then $A^{\text {out }}$ is hermitian.

c) For arbitrary $F \in \mathscr{F}\left(O_{+}\right)$one has $F \cdot D\left(A^{\text {out }}\right) \in D\left(A^{\text {out }}\right)$ and $\left[A^{\text {out }}, F\right] \Phi=0$ for any $\Phi \in D\left(A^{\text {out }}\right)$. An analogous statement holds for $A^{\text {out* }}$.

1) As in Ref.[1] we call the positive cone $\boldsymbol{O}_{+}$of all points which have a positive timelike separation from $\boldsymbol{O}$ the future tangent of $\boldsymbol{O}$. 


\section{Proof:}

a) We have already seen that $A^{\text {out }}$ is densely defined on the vectors $F \Omega, F \in \mathcal{F}\left(\sigma_{+}\right)$. The fact that it is closable follows immediately from the relation

$$
\begin{aligned}
& \left(F^{\prime} \Omega, A^{\text {out }} F \Omega\right)= \\
& =\lim _{T \rightarrow \infty}\left(F^{\prime} \Omega, A_{T} F \Omega\right)=\lim _{T \rightarrow \infty}\left(F^{\prime} A_{T}^{*} \Omega, F \Omega\right)=\left(F^{\prime} P_{1} A^{*} \Omega, F \Omega\right) \\
& \text { which holds for arbitrary } F, F^{\prime} \in \mathcal{F}\left(G_{+}\right) .
\end{aligned}
$$

b) This statement is a consequence of the above relation if one replaces. A by $A^{*}$.

c) For any $\Phi \in D\left(A^{\text {aut }}\right)$ there exists a sequence $F_{n} \in \mathcal{F}\left(G_{+}\right)$such that $s-\lim _{n} F_{n} \Omega=\Phi$ and $s-\lim _{n} A^{\text {out }} F_{n} \Omega=A^{\text {out }} \Phi$. Hence if $F \in \mathcal{F}\left(G_{+}\right)$

$$
s-\lim _{n} A^{\text {out }} F F_{n} \Omega=s-\lim _{n} F F_{n} \cdot P_{1} A \Omega=s-\lim _{n} F A^{\text {out }} F_{n} \Omega=F A^{\text {out }} \Phi
$$

and since $A^{\text {out }}$ is closed and $\underset{n}{\lim } F F_{n} \Omega=F \Phi$ the statement follows.

\section{Remark:}

Since the operator $A^{\text {out }}$ is only defined on the closure of $\left\{F \Omega: F \in \mathcal{F}\left(O_{+}\right)\right\}$ with respect to the graph topology it might seem to be necessary to lable $A^{\text {out }}$ also by the localisation region $O$ of $A$ (which was not unambiguously defined by the requirement that $A$ is an element of the algebra $\mathscr{F}(G)$ ). However we shall see later that $A^{\text {out }}$ does not depend on the precise shape of $G$.

So far the construction of the asymptotic fields $A^{\text {out }}$ did not differ very much from that of Ref.[1]. However, in order to verify that the unbounded operators $A^{\text {out }}$ may be used to build up the collision states we have to go now into a detailed analysis of their domains $D\left(A^{\text {out }}\right)$. The subsequent lemma will be an important tool in these investigations. In the formulation of this proposition we have to pay attention to the momentum space behaviour of the operators in $\mathfrak{F}_{0}$. For this purpose we distinguish a family of subsets $\mathcal{F}_{N}, N \in \mathbb{N}$ in $\mathfrak{F}_{0}$. The elenents of $\mathfrak{F}_{N}$ are all finite sums of operators of the form 
$\int d t \varphi(t) e^{-i t\left(P_{n}\right)} A e^{-i t\left(P_{n}\right)}, A \in \mathcal{F}_{0}$
where $\quad\left(P_{n}\right)=H n_{0}-\left(\underline{\left.P_{n}\right)}\right.$ is the component of the 4-momentum operator
$P \quad$ in the positive timelike direction with compact support which has a Fourier transform $\tilde{\varphi}(\omega)$ with an N-fold zero at $\omega=0$. So with increasing $N$ the operators in $\mathfrak{F}_{N}$ behave more and more smoothly at the origin in momentum space. Each $\mathcal{F}_{N}$ is a linear space of operators which is stable under taking adjoints and which is invariant under Poincaré transformations. Moreover, it maps the vacuum into a dense set of vectors in $\mathcal{H} \theta \Omega$. After these preliminary remarks we are prepared to formulate the proposition. The proof is given in the Appendix.

\section{Lemma 2:}

Let $A_{1}, \ldots A_{n}$ be elements of $\mathfrak{F}_{N}, N$ sufficiently large (depending on the total number $n$ of operators). Then

a) $\left\|A_{1 T} \cdots A_{n T} \Omega\right\| \leq c$ uniformly in $T$.

b) $\lim _{T \rightarrow \pm \infty}\left(\Omega, A_{1 T} \cdots A_{n T} \Omega\right)=\sum\left(\Omega, A_{i_{1}} P_{1} A_{i_{2}} \Omega\right) \cdots\left(\Omega, A_{i_{n-1}} P_{1} A_{i_{n}} \Omega\right)$

if $\mathrm{n}$ is even. The sum extends over all ordered pairs out of $(1 \ldots n)$. For odd $n$ the limit vanishes.

The fact that the sequences $A_{1 T} \cdots A_{n T} \Omega$ are uniformly bounded in $T$ will enable us to establish their convergence in the limit of large $T$. There is no reason to doubt that the limit vectors $\Psi^{\text {out }}\left(A_{1}, \ldots A_{n}\right)$ are just the collision states of massless Bosons we are interested in. However in order to verify this we need some more informations. It will be important for our argument that there exists an alternative way of constructing the vectors $\Psi^{\text {out }}\left(A_{1}, \ldots A_{n}\right)$ with the aid of the asymptotic fields: $\Psi^{\text {out }}\left(A_{1}, \ldots A_{n}\right)=A_{1}^{\text {out }} \ldots A_{n}^{\text {out }} \Omega$. We give the precise statements in the following lemma.

Lemma 3:

Let $A, A_{1}, \ldots A_{n}$ be elements of $\mathfrak{F}_{N}, N$ sufficiently large.

a) Then the weak limit

$$
\omega-\lim _{T \rightarrow \infty} A_{1 T} \cdots A_{n T} \Omega=\Psi^{\text {out }}\left(A_{1}, \ldots A_{n}\right)
$$


exists. It is multilinear in $A_{1}, \ldots A_{n}$ and depends only on the oneparticle states $P_{1} A_{1} \Omega, \ldots P_{1} A_{n} \Omega$.

b) $\Psi^{\text {out }}\left(A_{1}, \ldots A_{n}\right)$ is in the domain of $A^{\text {out* }}$ and

$$
A^{\text {out * }} \Psi^{\text {out }}\left(A_{1}, \ldots A_{n}\right)=\Psi^{\text {out }}\left(A^{*}, A_{1}, \ldots A_{n}\right)
$$

c) If in addition $A$ is localized in $\mathcal{O}$ and $A_{1}, \ldots A_{n}$ are localized in the future tangent $\mathcal{O}_{+}$of $\mathcal{O}$, then $\Psi^{\text {out }}\left(A_{1}, \ldots A_{n}\right)$ is also in the domain of $\mathrm{A}^{\text {out }}$ and

$$
A^{\text {out }} \Psi^{\text {out }}\left(A_{1}, \ldots A_{n}\right)=\Psi^{\text {out }}\left(A, A_{1}, \ldots A_{n}\right) .
$$

Proof:

a) We give a proof by induction: for $n=1$ the statement follows from relation (8). So let us assume that it holds for $(n-1)$. Now if $A_{1}$ is localized in $\mathcal{O}$ we get for any $F \in \mathcal{F}\left(\mathcal{O}_{+}\right)$

$$
\begin{aligned}
& \lim _{T \rightarrow \infty}\left(F \Omega, A_{1 T} \cdots A_{n T} \Omega\right)= \\
= & \lim _{T \rightarrow \infty}\left(F A_{1 T}{ }^{*} \Omega, A_{2 T} \cdots A_{n T} \Omega\right)=\lim _{T \rightarrow \infty}\left(F P_{1} A_{1}^{*} \Omega, A_{2 T} \cdots A_{n T} \Omega\right)=\left(F P_{1} A_{1}^{*} \Omega, \Psi^{\text {out }}\left(A_{2} \cdots A_{n}\right)\right)
\end{aligned}
$$
where we made use of the fact that $A_{1 T}{ }^{*} \Omega$ converges strongly and $A_{2 T} \cdots A_{n T} \Omega \quad$ weakly (by assumption). Thus the sequence $A_{1 \boldsymbol{n}} \cdots A_{n \boldsymbol{n}} S L$ converges on the dense set of vectors $F \Omega, F \in \mathcal{F}\left(O_{+}\right)$and since it is uniformly bounded (Lemma 2) it converges weak1y.

The statement concerning the linear properties of $\Psi^{\text {out }}\left(A_{1}, \ldots A_{n}\right)$ needs no extra explanation. In order to verify that the vectors depend only on the one-particle states $P_{1} A_{1} \Omega, \ldots P_{1} A_{n} \Omega$ we observe that the relation $P_{1} A \Omega=0$ implies $A^{\text {out }}=0$ and therefore also $\left(A^{* \text { out }}\right)^{*}=0$, because $\left(A^{* \text { out }}\right)^{*}$ is an extension of the closed operator $A^{\text {out }}$ (Lemma 1). Therefore, anticipating part $b$ ) of the lemma we get

$$
\Psi^{\text {out }}\left(A_{1}, \ldots A_{n}\right)=\left(A_{1}^{* \text { out }}\right)^{*} \ldots\left(A_{n}^{* \text { out }}\right)^{*} \Omega=0
$$

if any one of the operators $A_{i}$ maps the vacuum $\Omega$ into the orthogonal complement of $H_{1}: P_{1} A_{i} \Omega=0$.

b) This statement follows from

$$
\begin{aligned}
& \left(A^{\text {out }} F \Omega, \Psi^{\text {out }}\left(A_{1}, \ldots A_{n}\right)\right)= \\
= & \lim _{T \rightarrow \infty}\left(A_{T} F \Omega, A_{1 T} \cdots A_{n T} \Omega\right)=\lim _{T \rightarrow \infty}\left(F \Omega, A_{T}^{*} A_{1 T} \cdots A_{n T} \Omega\right)=\left(F \Omega, \Psi^{\text {out }}\left(A^{*}, A_{1}, \cdots A_{n}\right)\right),
\end{aligned}
$$


bearing in mind that the set $\left\{F \Omega: F \in F\left(G_{+}\right)\right\}$is a core for the operator $A^{\text {out }}$ by its very definition.

c) If $\Phi$ is any vector in the domain of $A^{\text {out * }}$ we get

$$
\left(A^{\text {out }} * \Phi, \Psi^{\text {out }}\left(A_{1}, \ldots A_{n}\right)\right)=\lim _{T \rightarrow \infty}\left(\Phi, A^{\text {out }} A_{1 T} \cdots A_{n T} \Omega\right)
$$

because the vectors $A_{1} \cdots A_{m} \Omega$ are (for large $T$ ) in the domain of $A^{\text {out }}$ owing to the localisation properties of $A_{1}, \ldots A_{n}$. So we have only to verify that the weak limit $\omega-\lim _{r \rightarrow \infty} A^{\text {out }} A_{1 T} \cdots A_{n r} \Omega \quad$ exists. Now

$$
\begin{aligned}
& \left\|A^{\text {out }} A_{1 T} \cdots A_{n T} \Omega\right\|^{2}=\left(A^{\text {out }} A_{1 T} \cdots A_{n T} \Omega, A^{\text {out }} A_{1 T} \cdots A_{n T} \Omega\right)= \\
= & \left(A^{\text {out }} A_{n T}^{*} \cdots A_{1 T}^{*} A_{1 T} \cdots A_{n T} \Omega, A^{\text {out }} \Omega\right)=\left(A_{n T}^{*} \cdots A_{1 T}^{*} A_{1 T} \cdots A_{n T} \Omega, A^{\text {out }} * A^{\text {out }} \Omega\right) \\
\leq & \left\|A_{n T}^{*} \cdots A_{1 T}^{*} A_{1 T} \cdots A_{n T} \Omega\right\| \cdot\left\|A^{\text {out } *} A^{\text {out }} \Omega\right\| \leq C
\end{aligned}
$$

uniformly in T. ( Here we used part c) of Lema 1, Lemma 2 and part b) of the present proposition). Therefore it suffices again to establish the convergence of the sequence $A^{\text {out }} A_{1 T} \cdots A_{n T} \Omega$ on the dense set of vectors $F \Omega, F \in \mathcal{F}\left(G_{+}\right)$. But

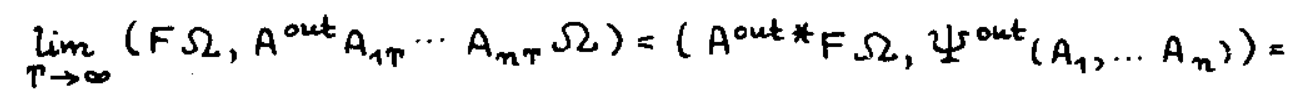

$=\left(A^{*}\right.$ out $\left.F \Omega, \Psi^{\text {out }}\left(A_{1}, \ldots A_{n}\right)\right)=\left(F \Omega,\left(A^{* \text { out }}\right)^{*} \Psi\left(A_{1}, \ldots A_{n}\right)\right)=\left(F \Omega, \Psi^{\text {out }}\left(A, A_{1}, \ldots A_{n}\right)\right)$ and this completes the proof of the lemma.

It needs no extra explanation that the whole construction can be carried out equally well at large negative times $T$. Since the results are completely analogous it is not necessary to $l$ ist them here.

\section{The Collision States}

We take a break now in our discussion of the asymptotic fields to analyse the vectors $\Psi^{\text {out }}\left(A_{1}, \ldots A_{n}\right)$. As was indicated above it will turn out that these vectors are just the collision states of massless Bosons. To begin with we show that they have the correct scalar products.

\section{Lemma 4:}

Let $A_{1}^{*}, \ldots A_{m}^{*}, A_{m+1}, \ldots A_{n}$ be elements of $\mathfrak{F}_{N}, N$ sufficiently large. If the first $m$ operators $A_{1}^{*}, \ldots A_{m}^{*}$ are localized in regions $\sigma_{1}, \ldots G_{m}$ 
with a positive timelike separation, $\quad \boldsymbol{V}_{i} \subset\left(\boldsymbol{O}_{i+1}\right)+$ for $i=1, \ldots \mathrm{m}-1$, then

$$
\left(\Psi^{\text {out }}\left(A_{m}^{*}, \ldots A_{1}^{*}\right), \Psi^{\text {out }}\left(A_{m+1}, \ldots A_{n}\right)\right)=\sum\left(\Omega L, A_{i_{1}} P_{1} A_{i_{2}} \Omega l\right) \cdots\left(\Omega, \hat{A}_{i_{n-1}} P_{1} A_{i_{n}} \delta \Omega\right)
$$

if $n$ is even. The sum extends over all ordered pairs out of $(1, \ldots n)$. For odd $n$ the scalar product vanishes.

\section{Proof:}

Owing to the localisation properties of $A_{1}^{*}, \ldots A_{m}^{*}$ we can apply part $b$ ) and $c$ ) of Lemma 3 and write

$$
\begin{aligned}
& \left(\Psi^{\text {out }}\left(A_{m}^{*}, \ldots A_{1}^{*}\right), \Psi^{\text {out }}\left(A_{m+1}, \ldots A_{n}\right)\right)=\left(A_{m}^{* \text { out }} \ldots A_{1}^{\text {*out }} \Omega, \Psi^{\text {out }}\left(A_{m+1}, \ldots A_{m}\right)\right)= \\
= & \left(\Omega,\left(A_{1}^{* \text { out }}\right)^{*} \ldots\left(A_{m}^{* \text { out }}\right)^{*} \Psi^{\text {out }}\left(A_{m+1}, \ldots A_{n}\right)\right)=\left(\Omega, \Psi^{\text {out }}\left(A_{1}, \ldots A_{n}\right)\right) .
\end{aligned}
$$

On the other hand we have $\Psi^{\text {out }}\left(A_{1}, \ldots A_{n}\right)=\omega-\lim _{T \rightarrow \infty} A_{1 \uparrow} \cdots A_{n r} \Omega$.

We get therefore from part $b$ ) of Lemma 2

$$
\left(\Omega, \Psi^{\text {out }}\left(A_{1}, \ldots A_{n}\right)\right)=\sum\left(\Omega, A_{i_{1}} P_{1} A_{i_{2}} \Omega\right) \cdots\left(\Omega, A_{i_{n-1}} P_{1} A_{i_{n}} \Omega\right)
$$

if $\mathrm{n}$ is even and zero if $\mathrm{n}$ is odd. This completes the proof.

In order to extend this result to arbitrary configurations of operators $A_{1}, \ldots A_{n}$ it is convenient to distinguish suitable linear combinations of the vectors $\Psi^{\text {out }}\left(A_{1}, \ldots A_{n}\right)$. For any given set of operators $A_{1}, \ldots A_{n} \in F_{N}$, $\mathrm{N}$ sufficiently large, we define recursively

$$
\begin{aligned}
& \Phi_{i}=\left(A_{i}^{* \text { out }}\right)^{*} \Omega=P_{1} A_{i} \Omega \\
& \Phi_{i}{ }^{\text {out }} \Phi_{j}=\left(A_{i}^{* \text { out }}\right)^{*} \Phi_{j}-\left(\Omega, A_{i} P_{1} A_{j} \Omega\right) \cdot \Omega \\
& \Phi_{i_{1}}^{\text {out }} \Phi_{i_{2}}^{\text {out }} x^{\text {out }} \Phi_{i_{n}}=\left(A_{i_{1}}^{* \text { out }}\right)^{*} \cdot \Phi_{i_{2}}^{\text {out } \cdots x} \Phi_{i_{n}}-\sum_{k=2}^{n}\left(\Omega, A_{i_{1}} P_{1} A_{i_{k}} \Omega\right) \cdot \Phi_{i_{2}}^{\text {out }} \cdots V^{i_{k}} \cdots x^{\text {out }} \Phi_{i_{n}}
\end{aligned}
$$
nothing else but normal ordering; proceeding from $\Psi^{\text {out }}\left(A_{1}, \ldots A_{n}\right)$ to $\Phi_{1} \times{ }^{\text {out }} \ldots{ }^{\text {out }} \times \Phi_{n}$ amounts to subtracting from $\Psi$ out $\left(A_{1}, \ldots A_{n}\right)$ all contributions with a particle number less than $n$. The labeling of the normal ordered vectors $\Phi_{1}^{\text {out }} \times{ }^{\text {out }} \Phi_{n}$ by the one-particle states $\Phi_{i}=P_{1} A_{i} \Omega$ is justified by the fact that the vectors $\Psi^{\text {out }}\left(A_{1}, \ldots A_{n}\right)$ depend in a linear way on $P_{1} A_{i} \Omega$ 
according to Lemma 3. It follows now immediately from Lemma 4 that for any collection of operators $A_{1}, \ldots A_{m} \in \mathcal{F}_{N}$ which are localized in regions $\boldsymbol{O}_{1}, \ldots \boldsymbol{O}_{\boldsymbol{m}}$ with a positive timelike separation, $\boldsymbol{O}_{i} \subset\left(\boldsymbol{O}_{i-1}\right)_{+}, i=2, \ldots m$ and any other collection $A_{1}^{\prime}, \ldots A_{n}^{\prime} \in \mathcal{F}_{N}$ with no requirements on the $10 \mathrm{cal} i-$ sation regions, (in an obvious notation)

$$
\left(\Phi_{1}^{\text {out }} \cdots \stackrel{\text { out }}{x} \Phi_{m}, \Phi_{1}^{\text {out }}{ }_{x}^{\text {out }} \phi_{x}^{\prime}\right)=\delta_{m n} \cdot \sum_{p}\left(\Phi_{1}, \Phi_{p(1)}^{\prime}\right) \cdots\left(\Phi_{n}, \Phi_{p(n)}^{\prime}\right)
$$

if $\mathrm{N}$ is sufficiently large. The sum extends over all permutations $P=$ $(p(1), \ldots p(n))$ of $(1, \ldots n)$. In particular one gets for the restricted class of vectors

$$
\left\|\Phi_{1} \stackrel{\text { out }}{x} \cdot \stackrel{\text { out }}{x} \Phi_{m}\right\|^{2} \leq m ! \cdot\left\|\Phi_{1}\right\|^{2} \cdots\left\|\Phi_{m}\right\|^{2}
$$

and this shows that these vectors depend continuously on the one-particle states $\Phi_{i}$.

Now for any set of vectors $\widehat{\Phi}_{1}, \ldots \widehat{\Phi}_{m} \in \mathcal{X}_{1}$ one can specify sequences of operators $A_{1}^{(n)}, \ldots A_{m}^{(n)} \in \mathcal{F}_{N} \quad$ with localisation properties given above, such that simultaneously for all $i=1, \ldots m$ the strong limits $s-\lim _{n} P_{1} A_{i}^{(n)} \Omega=\hat{\Phi}_{i}$ exist. ${ }^{2)}$ So the continuity of the vectors $\Phi_{1}^{\text {out }} \times{ }^{\text {out }} \Phi_{m}$ with respect to the one-particle constituents $\Phi_{i}$ makes an extension possible to arbitrary configurations $\widehat{\Phi}_{1}, \ldots \hat{\Phi}_{m} \in \mathscr{K}_{1}$. We use the symbo1 $\hat{\Phi}_{1} \times \ldots \times$ out $\hat{\Phi}_{m}$ also for these extensions. This is a consistent notation because the restricted class of vectors is dense in the set of vectors which were defined by relation (11) for arbitrary operators $A_{1}, \ldots A_{m} \in F_{N}$ (and not only for the particular configurations considered above). For a proof

2) It follows from a Reeh-Schlieder type argument given in Ref.[1], that the operators $F \in \mathfrak{F}_{N}$ which are localized in the future tangent of some bounded region 6 generate from the vacuum a dense set of vectors in $\left[\mathscr{F}_{N} \Omega\right]=\mathcal{H} \Omega$. Hence, given $\mathrm{n}$, one can find a bounded region $G_{1}^{(n)}$ and an operator $A_{1}^{(n)} \in \widetilde{F}_{N}$ which is localized in $G_{1}^{(n)}$ such that $\left\|\hat{\Phi}_{1}-P_{1} A_{1}^{(n)} \Omega\right\| \leq n^{-1}$. Then there exist another bounded region $G_{2}^{(n)}$ in the future tangent of $\sigma_{1}^{(n)}$ and an operator $A_{2}^{(n)}$ which is localized in $G_{2}^{(n)}$ such that $\left\|\hat{\Phi}_{2}-P_{1} A_{2}^{(n)} \Omega\right\| \leq n^{-1}$ and so on. 
of this statement we recall that the weak convergence of a sequence $\Psi_{n}$ implies $\left\|w-\lim _{n} \Psi_{n}\right\| \leq \lim _{n}\left\|\Psi_{n}\right\|$ and this fact together with part a) of Lemma 3 and part b) of Lemma 2 allows to show

$$
\left\|\Phi_{1}^{\text {out }} \times{ }_{x}^{\text {out }} \Phi_{m}\right\|^{2} \leq \sum_{P}\left(\Phi_{1}, \Phi_{p(1)}\right) \cdots\left(\Phi_{n}, \Phi_{p(n)}\right)
$$

for arbitrary operators $A_{1}, \ldots A_{m} \in \mathfrak{F}_{N}$. If we take now (as above)

sequences of operators $A_{1}^{(n)}, \ldots A_{m}^{(n)} \in \mathcal{F}_{N}$ such that the vectors $\Phi_{i}^{(n)}=P_{1} A_{i}^{(n)} \Omega$ converge to $\Phi_{i}=P_{1} A_{i} \Omega$, then the sequence $\Phi_{1}^{(n)} x^{\text {out }} \ldots x^{\text {out }} \Phi_{m}^{(n)}$ converges to some vector $\Psi$. Hence using relation (12) and (14) we get the estimate

$\left(\Psi, \Phi_{1}^{\text {out }} \times \cdots \times \Phi_{m}^{\text {out }}\right)=\sum_{p}\left(\Phi_{1}, \Phi_{p(1)}\right) \cdots\left(\Phi_{m}, \Phi_{p(m)}\right)=\|\Psi\|^{2} \geq\|\Psi\|\left\|\Phi_{1}^{\text {out }} \cdots x^{\text {out }} \Phi_{m}\right\|$. (15) This is however incompatible with Cauchy's inequality unless $\Psi=\Phi_{\mathbf{1}}^{\text {out }} \cdots \times \boldsymbol{x}_{\mathbf{m}}$. It needs now no extra explanation that for arbitrary $\Phi_{1}, \ldots \Phi_{m} \in \mathcal{H}_{1}$ the vectors $\Phi_{1} \times x^{\text {out }} \ldots \stackrel{\text { out }}{x} \Phi_{m}-$ and thus a fortiori the vectors defined in relation (11) -- have the scalar products given by relation (12).

\section{Remark:}

Knowing the scalar products of $\Phi_{1}{ }_{x}^{\text {out }} \ldots{ }^{\text {out }} \times \Phi_{m}$ and therefore also those of $\Psi^{\text {out }}\left(A_{1}, \ldots A_{m}\right) \quad$ it is obvious that the sequences $A_{1} \cdots A_{m} \boldsymbol{\Omega}$ defined in Lemma 3 converge strongly: they converge weakly and in addition $\left\|\omega-\lim _{T \rightarrow \infty} A_{1 T} \cdots A_{m T} \Omega\right\|=\lim _{T \rightarrow \infty}\left\|A_{1 T} \cdots A_{m T} \Omega\right\|$.

Our next task consists in checking the transformation properties of $\Phi_{\mathbf{1}}^{\text {out }} \ldots \mathbf{x u t}_{\mathbf{x}} \mathbf{\Phi}_{\mathbf{m}}$ under Poincaré transformations $U(L), L \in P$. As expected we get for any set of vectors $\Phi_{1}, \ldots \Phi_{m} \in \mathscr{Z}_{1}$

$$
U(L) \cdot \Phi_{1}^{\text {out }} \ldots{ }^{\text {out }} \times \Phi_{m}=\left(U_{1}(L) \Phi_{1}\right)_{x}^{\text {out }} \ldots \text { out } \times\left(U_{1}(L) \Phi_{m}\right)
$$

where $L \rightarrow u_{\mathbf{1}}(L)$ denotes the representation of the poincaré group $\mathcal{P}$ in $\mathfrak{K}_{\mathbf{1}}$. In order to verify equation (16) it suffices to prove

$$
U(L) \Psi^{\text {out }}\left(A_{1}, \ldots A_{m}\right)=\Psi^{\text {out }}\left(\alpha_{L}\left(A_{1}\right), \ldots \alpha_{L}\left(A_{m}\right)\right)
$$

for arbitrary operators $A_{1}, \ldots A_{m} \in \mathfrak{F}_{N}$. We shall do this by induction. 
For $n=1$ the statement holds trivially. So assuming that it holds for $(n-1)$ we get the string of equations (using Lemma 3)

$$
\begin{aligned}
& \left(F \Omega, U(L) \Psi^{\text {out }}\left(A_{1}, \ldots A_{m}\right)\right)=\left(\alpha_{L-1}(F) \Omega, \Psi^{\text {out }}\left(A_{1}, \ldots A_{m}\right)\right)= \\
= & \left(\alpha_{L-1}(F) \Omega,\left(A_{1}^{* \text { out }}\right)^{*} \Psi^{\text {out }}\left(A_{2}, \ldots A_{m}\right)\right)=\left(\alpha_{L-1}(F) P_{1} A_{1}^{*} \Omega, \Psi^{\text {out }}\left(A_{2}, \ldots A_{m}\right)\right) * \\
= & \left(F P_{1} \alpha_{L}\left(A_{1}^{*}\right) \Omega, U(L) \Psi^{\text {out }}\left(A_{2}, \ldots A_{m}\right)\right)=\left(\alpha_{L}\left(A_{1}^{*}\right)^{\text {out }} F \Omega, \Psi^{\text {out }}\left(\alpha_{L}\left(A_{2}\right), \ldots \alpha_{L}\left(A_{m}\right)\right)\right)= \\
= & \left(F \Omega, \Psi^{\text {out }}\left(\alpha_{L}\left(A_{1}\right), \ldots \alpha_{L}\left(A_{m}\right)\right)\right)
\end{aligned}
$$

as long as $F$ is localized in the future tangent of the localisation region of $\alpha_{L}\left(A_{1}^{*}\right)$. This establishes relation (17).

Finally we mention that the vectors $\Phi_{1} x^{\text {out }} \ldots \stackrel{\text { out }}{x} \Phi_{m}$ are symmetric under permutations of the one-particle constituents $\Phi_{i}:$ if $(p(1), \ldots p(m))$ is any permutation of the numbers $(1, \ldots m)$ then it follows from the symmetry properties of the scalar products given in relation (12) that

$$
\Phi_{p(1)}^{\text {out }} \times \text { out } \Phi_{p(m)}=\Phi_{1}^{\text {out }} \ldots \text { out } x \Phi_{m} \text {. }
$$

So the massless particles in oux model really obey Bose-statistics. Summing up we realize that the vectors $\Phi_{1}^{\text {out }}{ }^{\text {out }}{ }_{x} \Phi_{n}$ have the features expected from an asymptotically freely moving configuration of massless particles $\Phi_{1}, \ldots \Phi_{n}$. We compile the most relevant properties of these vectors in the following theorem.

\section{Theorem 5:}

Let $\Phi_{1}, \ldots \Phi_{n} \in \mathcal{H}_{1}$ be any collection of massless one-particle states. Then the vectors $\Phi_{1} \times{ }^{\text {out }} \ldots{ }_{x}^{\text {out }} \Phi_{m}$ defined above have the following properties:
a) $\Phi_{p(1)}$ out $^{\text {out... out }} \Phi_{p(n)}=\Phi_{1}^{\text {out }} \ldots \Phi_{x}^{\text {out }} \Phi_{n}$ for any permutation
$P=(p(1), \ldots p(n)) \quad$ of the numbers $(1, \ldots, n)$.

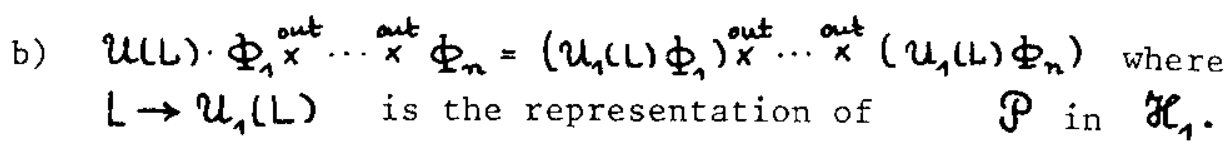

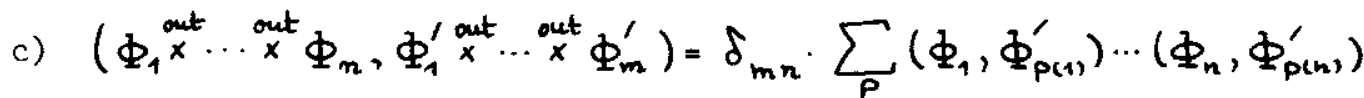 and the sum extends over al1 permutations $P$ of $(1, \ldots n)$. 
So the Hilbert space $\mathcal{H}^{\text {out }}$ which is generated by $\Omega$ and the vectors $\Phi_{1} x^{\text {out }} \ldots{ }^{\text {out }} \Phi_{n}, n \in \mathbb{N}$ is the familiar Fock-space over the one-particle space $\mathcal{H}_{1}$. It is obvious from our construction that the vectors in this space may be interpreted (in terms of measurements at large positive times) as outgoing configurations of massless particles. Therefore the usual definition and interpretation of a scattering matrix for the massless particles is possible and makes physical sense.

\section{The Asymptotic Field Algebras}

So far the asymptotic fields $A^{\text {out }}$ have served as an aid for the construction of the collision states. However, from the point of view of physics their significance should go much beyond that: in quantum electrodynamics for example, one expects that the operators $\mathrm{A}^{\text {out }}$ correspond tofield strength measurements performed at large positive times; similar interpretations in terms of observables should be possible in other models. Now it is one of the basic principles in quantum mechanics that an operator has to admit a spectral decomposition in order to be accepted as an observable. It is therefore gratifying that one can specify within our general framework a large set of selfadjoint asymptotic field operators $A^{\text {out }}$. The proof of this assertion is based on the subsequent lenma in which it is shown that the operators $A^{\text {out }}$ act on the collision states $\Phi_{1} \times$ out $\ldots \times \Phi_{n}$ like a free field.

\section{Lemma 6:}

Let $A$ be an element of $\mathfrak{F}_{N_{0}}, N_{0}$ sufficiently large. Then the collision states $\Phi_{1} \times{ }^{\text {aut.... out }} \Phi_{n}$ are in the domain of $A^{\text {out }}$ for arbitrary configurations $\Phi_{1}, \ldots \Phi_{n} \in \mathcal{H}_{1}$ and every $n \in \mathbb{N}$. Furthermore,

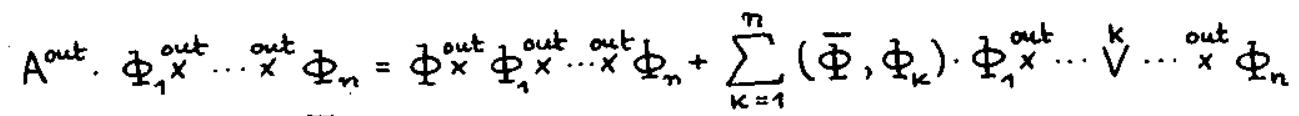

where $\Phi=P_{1} A \Omega$ and $\Phi=P_{1} A^{*} \Omega$.

\section{Proof:}

Let the operators $A_{1}, \ldots A_{n} \in \mathcal{F}_{N}$ be localized in the future tangent $\sigma_{+}$of the localisation region $O$ of $A \in \mathcal{F}_{N}$. Then it is evident from relation (11), part b) of Lemma 1 and part C) of Lemma 3 that the statement holds for the configuration $\Phi_{1}=P_{1} A_{1} \Omega, \ldots \Phi_{n}=P_{1} A_{n} \Omega$, provided $N$ is big enough (depending on $n$ ). We shall extend this result to the operators $A^{\text {out }}, A \in \mathcal{F}_{N_{0}}$, where $N_{0}$ 
is some fixed, sufficiently large number which does not depend on $n$. For this purpose we recapitulate the proof of part c) of Lemma 3: if $\Phi \in D\left(A^{\text {out }} *\right)$, then

$$
\left|\left(A^{\text {out } *} \Phi, \Psi^{\text {out }}\left(A_{1}, \ldots A_{n}\right)\right)\right|=\left|\lim _{T \rightarrow \infty}\left(\Phi, A^{\text {out }} A_{1 T} \ldots A_{n T} \Omega\right)\right| \leq \lim _{T \rightarrow \infty} \sup _{T \rightarrow \infty}\|\cdot\| A^{\text {out }} A_{1 T} \cdots A_{n T} \Omega \| .
$$

Now owing to the localisation properties of $A_{1}, \ldots A_{n}$ we get

$$
\left\|A^{\text {out }} A_{1 T} \cdots A_{n T} \Omega\right\|^{2} \leq\left\|A_{n T}{ }^{*} \cdots A_{1 T}{ }^{*} A_{1 T} \cdots A_{n T} \Omega\right\| \cdot\left\|A^{\text {out }} * A^{\text {out }} \Omega\right\| .
$$

This expression is uniformly bounded if $A_{1}, \ldots A_{n} \in \mathscr{F}_{N}, N$ sufficiently large, and $A \in \mathcal{F}_{N_{0}}$ where $N_{0}$ is such that $\left\|A^{\text {out } *} A^{\text {out }} \Omega\right\|<\infty$. 3) Hence the vectors $\Psi^{\text {out }}\left(A_{1}, \ldots A_{n}\right)$, and therefore also $\Phi_{1}^{\text {out }} \times$ out $x \Phi_{n}$, are elements of $D\left(A^{\text {out }}\right)$. Next we calculate how $A^{\text {out }}$ acts on these vectors. For this purpose we take a sequence $A^{(m)} \in F_{N}$ such that $s-\lim _{m} P_{1} A^{(m)} \Omega=P_{1} A \Omega$ and $s-\lim _{m} P_{1} A^{(m) *} \Omega=P_{1} A^{*} \Omega$; we require furthermore that the operators $A^{(m)}$ are localized in the timelike cylinder $\bigcup_{t \leq 0}^{m}\{\mathcal{O}+(t, \underline{0})\}$. A simple example reconciling these properties is

$$
A^{(m)}=m^{-1} \cdot \int_{-m}^{0} d t\left(1-\alpha_{t}\right)^{N}(A)
$$

where $\iota$ denotes the identity automorphism and $\boldsymbol{\alpha}_{t}$ the time translations.

Bearing in mind that the statement of the lemma has already been proven for the special configurations of operators $A, A_{1}, \ldots A_{n} \in \mathfrak{F}_{N}$ mentioned above we get for any $F \in \mathcal{F}\left(\boldsymbol{O}_{+}\right)$:

$$
\begin{aligned}
& \left(F \Omega, A^{\text {out }} \Phi_{1}^{\text {out }} \ldots{ }^{\text {out }} \Phi_{n}\right)=\left(F P_{1} A^{*} \Omega, \Phi_{1}^{\text {out }} \ldots{ }^{\text {out }} \Phi_{n}\right)= \\
& =\lim _{m}\left(F P_{1} A^{(m) *} \Omega, \Phi_{1}^{\text {out }} \ldots \text { out } \Phi_{n}\right)=\lim _{m}\left(F \Omega, A^{(m) \text { out }} \Phi_{1}^{\text {out }} \times{ }^{\text {out }} \times \Phi_{n}\right)= \\
& =\lim _{m}\left\{\left(F \Omega, \Phi_{x}^{(m) \text { out }} \Phi_{1}^{\text {out }} \times \ldots{ }_{x}^{\text {out }} \Phi_{n}\right)+\sum_{k=1}^{m}\left(\overline{\Phi^{(m)}}, \Phi_{k}\right) \cdot\left(F \Omega, \Phi_{1}^{\text {out }} \ldots V^{k} \ldots{ }^{\text {out }} \Phi_{n}\right)\right\}= \\
& =\left(F \Omega,\left\{\Phi^{\text {out }} \Phi_{1}^{\text {out }} \times \cdots{ }^{\text {out }} \Phi_{n}+\sum_{k=1}^{n}\left(\Phi, \Phi_{k}\right) \cdot \Phi_{1}^{\text {out }} \times \cdots V^{k} \ldots \stackrel{\text { out }}{x} \Phi_{n}\right\}\right) \text {. }
\end{aligned}
$$

3) Since we want to apply the estimates given in the Appendix we have to choose $\mathrm{N}_{\mathrm{O}}=15$ here. However we conjecture that all propositions in this chapter remain true if $\mathrm{N}_{0}=0$. 
This proves the lemma for the special configurations; the extension to arbitrary configurations $\Phi_{1}, \ldots \Phi_{n} \in X_{1}$ can be performed owing to the continuity of the vectors $\Phi_{1}^{\text {out }} \ldots{ }_{x}^{\text {out }} \Phi_{n}$ with respect to the one-particle consituents and the fact that $A^{\text {out }}$ is closed.

That the operators $A^{\text {out }}$ act on the collision states like a free field will enable us to specify a dense set of analytic vectors in their domain. It follows then from a well known theorem of Nelson [8; Theorem X.39] that A is selfadjoint provided it is hermitian.

\section{Theorem 7:}

Let $A=A^{*}$ be an element of $\mathfrak{F}_{N_{0}}, N_{0}$ as in Lema 6 .

a) Then $A^{\text {out }}$ is selfadjoint.

b) If $\boldsymbol{O}_{1}$ is a region with a non-empty future-tangent $\left(\boldsymbol{G}_{1}\right)_{+}$and if $\boldsymbol{G} \subset \boldsymbol{\sigma}_{1}$, then the dense set of vectors $\left\{F \Omega: F \in \mathcal{F}\left(\left(G_{1}\right)_{+}\right)\right\}$is a core for $A^{\text {out }}$.

c) $A^{\text {out }}$ is uniquely determined by the one-particle state $P_{1} A \Omega \in \mathcal{K}_{1}$ if A varies within the above restrictions.

\section{Proof:}

a) Using part c) of Lemna 1, Lema 6 and relation (12) it is straightforward to verify that

$$
\left\|\left(A^{\text {out }}\right)^{n} \cdot F \Omega\right\|^{2} \leq\|F\|^{2} \cdot\left\|\left(A^{\text {out }}\right)^{n} \Omega\right\|^{2}=\|F\|^{2} \cdot \frac{(2 n) !}{2^{n} \cdot n !} \cdot\left\|P_{1} A \Omega\right\|^{2 n}
$$

for arbitrary $F \in \mathfrak{F}\left(\mathcal{O}_{+}\right)$. This estimate shows that the dense set of vectors $\left\{F \Omega: F \in \mathfrak{F}\left(G_{+}\right)\right\}$is a set of analytic vectors (in the terminology of [8]) for the operator $A^{\text {out }}$. Since $A^{\text {out }}$ is hermitian (Lemma 1) the theorem of Nelson quoted above guarantees that $A^{\text {out }}$ is selfadjoint.

b) Since $G \subset \sigma_{1}$ and $\left(O_{1}\right)_{+}$is not empty we can restrict $A^{\text {out }}$ to the dense set of vectors $\left\{F \Omega: F \in \mathcal{F}\left(\left(G_{1}\right)_{+}\right)\right\}$. This restriction defines a closable operator and we denote its least closed extension by $\mathrm{A}^{\text {out }}$. Now the whole argument establishing the selfadjointness of $A^{\text {out }}$ can be applied likewise to $\overparen{A^{\text {out }}}$. Hence $\overparen{A^{\text {ut }}}$ is also selfadjoint. But a selfadjoint 
operator is maximal, and since $A^{\text {out }}$ is a selfadjoint extension of $\widehat{A}^{\text {out }}$ we get $\widehat{A^{\text {out }}}=A^{\text {out }}$.

c) Let $A_{1}, A_{2} \in \mathfrak{F}_{N_{0}} \quad$ be two selfadjoint operators satisfying $P_{1} A_{1} \Omega=P_{1} A_{2} \Omega$. Then there exists a bounded region $\mathcal{O}$, containing the localisation regions $\boldsymbol{O}_{1}$ and $\boldsymbol{O}_{2}$ of $A_{1}$ and $A_{2}$, such that the operators $A_{1}^{\text {out }}$ and $A_{2}^{\text {out }}$ coincide on the dense set $\left\{F \Omega: F \in \mathcal{F}\left(G_{+}\right)\right\}$. However these vectors are a core for both operators, according to part $b$ ) of the theorem, and therefore $A_{1}^{\text {out }}=A_{2}^{\text {out }}$.

With this theorem at our disposal it is now fairly simple to prove that the operators $A^{\text {out }}$ have all essential features of a free, massless field. But there is one little difference: it is in general not possible to specify a set of vectors in the domain of $A^{\text {out }}$, which is dense in $\mathscr{H}$ and invariant under arbitrary translations. Therefore the operator-valued function $x \rightarrow A^{\text {out }}(x)=U(x) A^{\text {out }} U(x)^{-1}$ cannot be defined globally. However if we confine our attention to open regions $\mathscr{S}_{\subset} \mathbb{R}^{4}$ with a non-empty future tangent $\boldsymbol{I}_{+}$, then the intersection of the domains $U(x) D\left(A^{\text {out }}\right), x \in \varphi$ contains the dense set of vectors $D_{\varphi}\left(A^{\text {out }}\right)=\left\{F \Omega: F \in \bigcap_{x \in \mathscr{\varphi}} \mathfrak{F}\left(G_{+}+x\right)\right\}$

and the functions $x \rightarrow A^{\text {out }}(x) \Phi, x \in \boldsymbol{\varphi}^{x \in \mathscr{P}}$ are defined for all $\Phi \in D_{\boldsymbol{\varphi}}\left(A^{\text {out }}\right)$. The next theorem shows that these functions are covariant solutions of the wave-equation.

\section{Theorem 8:}

Let A satisfy the assumptions of. Theorem 7. Then
a) $\square_{x} A^{\text {out }}(x)=0$ on $D_{\varphi}\left(A^{\text {out }}\right)$ for $x \in \varphi$.
b) $\alpha_{L}\left(A^{\text {out }}\right)=\left(\alpha_{L}(A)\right)^{\text {out }}$ for $L \in \mathcal{P}$.

\section{Proof:}

a) This follows immediately from the relation

$$
A^{\text {out }}(x) F \Omega=U(x) A^{\text {out }} \alpha_{-x}(F) \Omega=F \cdot U(x)^{-1} P_{1} A \Omega
$$


for $F \in \bigcap_{x \in \mathcal{Y}} \mathfrak{F}(0+x)$ and the fact that $A$ is continuously differentiable with respect to the translations.

b) The intersection of the domains $U(L) D\left(A^{\text {out }}\right)$ and $D\left(\alpha_{L}(A)^{\text {out }}\right)$ contains the dense set of vectors $\left\{F \Omega: F \in F\left((L G)_{+}\right)\right\}$on which the operators coincide. This set is a core for both operators and therefore the statement follows.

\section{Remark:}

Although the functions $\quad x \rightarrow A^{\text {out }}(x), x \in \varphi$ are solutions of the waveequation it is in general not possible to split the operators $A^{\text {out }}$ into a creation and an annihilation part. This can only be done if there exists a dense set of vectors in $\mathcal{H}$ on which the operator valued functions $t \rightarrow A^{\text {out }}((t, 0))$ are defined for all $t \in \mathbb{R}$ (in the sense of tempered distributions).

Finally we have to analyze the commutation properties of the operators $A^{\text {out }}$. As expected it turns out that

$$
\left[A_{1}^{\text {out }}, A_{2}^{\text {out }}\right]=\omega_{0}\left(\left[A_{1}^{\text {out }}, A_{2}^{\text {out }}\right]\right) \cdot 1
$$

and the commutator vanishes in particular for operators $A_{1}$, $A_{2}$ with spacelike or timelike separated localisation regions. In order to exclude all possible pathologies connected with the unboundedness of the operators $A_{1}^{\text {out }}$, $A_{2}^{\text {out }}$ we reformulate relation (23) in terms of the resolvents $R\left(A^{\text {out }}\right)=\left(i+A^{\text {out }}\right)^{-1}$.

\section{Theorem 9:}

Let $A_{1}, A_{2}$ satisfy the assumptions of Theorem 7 . Then

a) $\left[R\left(A_{1}^{\text {out }}\right), R\left(A_{2}^{\text {out }}\right)\right]=\omega_{0}\left(\left[A_{1}^{\text {out }}, A_{2}^{\text {out }}\right]\right) \cdot R\left(A_{1}^{\text {out }}\right) R\left(A_{2}^{\text {out }}\right)^{2} R\left(A_{1}^{\text {out }}\right)$.

b) If $A_{1}, A_{2}$ are localized in two spacelike separated double cones then $\left[R\left(A_{1}^{\text {out }}\right), R\left(A_{2}^{\text {out }}\right)\right]=0$.

c) If $A_{1}$ is localized in some region $O$, then for all $F \in \mathcal{F}\left(G_{+}\right)$ $\left[R\left(A_{4}^{\text {out }}\right), F\right]=0$. 


\section{Proof:}

a) It follows from the definition of the approximations $A_{T}$ given in Chapter 2 that $A_{T}$ is selfadjoint and $R\left(A_{T}\right)=\left(i+A_{r}\right)^{-1}$ is uniformly bounded in $T$ if $A=A^{*}$. Therefore we get for all $F \in \mathscr{F}\left(O_{+}\right)$

$$
s-\lim _{T \rightarrow \infty}\left(i+A_{r}\right)^{-1} \cdot\left(i+A^{\text {out }}\right) F \Omega=s-\lim _{T \rightarrow \infty}\left(i+A_{T}\right)^{-1} \cdot\left(i+A_{T}\right) F \Omega=F \Omega
$$

proving that $\left(i+A_{T}\right)^{-1}$ converges strongly on the range of (i+ $A^{\text {out }}$ ).

However this is the whole Hilbert space $H^{\circ}$ if $A^{\text {out }}$ is selfadjoint. Hence we can write, after a little algebra

$$
\begin{aligned}
& {\left[R\left(A_{1}^{\text {out }}\right), R\left(A_{2}^{\text {out }}\right)\right]=s-\lim _{T \rightarrow \infty}\left[R\left(A_{1 T}\right), R\left(A_{2 T}\right)\right]=} \\
= & s-\lim _{T \rightarrow \infty}\left\{R\left(A_{1 T}\right) R\left(A_{2 T}\right)^{2}\left[A_{2 T},\left[A_{1 T}, A_{2 T}\right]\right] R\left(A_{2 T}\right) R\left(A_{1 T}\right)+\right. \\
+ & \left.R\left(A_{1 T}\right) R\left(A_{2 T}\right)^{2} R\left(A_{1 T}\right)\left[A_{1 T},\left[A_{1 T}, A_{2 T}\right]\right] R\left(A_{1 T}\right)+R\left(A_{1 T}\right) R\left(A_{2 T}\right)^{2} R\left(A_{1 T}\right) \cdot\left[A_{1 T}, A_{2 T}\right]\right\} .
\end{aligned}
$$

Now the first two terms in the curly bracket do not give a contribution, because in the limit of large $T$ the double commutators

\section{$\left[A_{1 T},\left[A_{1 T}, A_{2 T}\right]\right]$ and $\left[A_{2 T},\left[A_{1 T}, A_{2 T}\right]\right]$}

vanish in the uniform topology. (See the Appendix). So if $A_{1}$ and $A_{2}$ are localized in some region $\mathcal{O}$, we get for all $F \in \mathfrak{F}\left({O_{+}}_{+}\right)$(using part a) of Lemma 3, relation (11) and Theorem 5)

$$
\begin{aligned}
& {\left[R\left(A_{1}^{\text {out }}\right), R\left(A_{2}^{\text {out }}\right)\right] F \Omega=s-\lim _{T \rightarrow \infty} R\left(A_{1 T}\right) R\left(A_{2 T}\right)^{2} R\left(A_{1 T}\right) \cdot F\left[A_{1 T}, A_{2 T}\right] \Omega=} \\
= & \omega_{T \rightarrow \infty}-\lim _{T \rightarrow \infty} R\left(A_{1}^{\text {out }}\right) R\left(A_{2}^{\text {out }}\right)^{2} R\left(A_{1}^{\text {out }}\right) \cdot F\left[A_{1 T}, A_{2 T}\right] \Omega= \\
= & \omega_{0}\left(\left[A_{1}^{\text {out }}, A_{2}^{\text {out }}\right]\right) \cdot R\left(A_{1}^{\text {out }}\right) R\left(A_{2}^{\text {out }}\right)^{2} R\left(A_{1}^{\text {out }}\right) \cdot F \Omega
\end{aligned}
$$

and this completes the proof of the first third of the lemma.

b) Owing to the preceding result it suffices to consider the expectation value

$$
\begin{aligned}
& \left(\Omega,\left[A_{1}^{\text {out }}, A_{2}^{\text {out }}\right] \Omega\right)= \\
& =\left(\Omega, A_{1} P_{1} A_{2} \Omega\right)-\left(\Omega, A_{2} P_{1} A_{1} \Omega\right)=\left(\Omega, A_{1} P(m=0) A_{2} \Omega\right)-\left(\Omega, A_{2} P(m=0) A_{1} \Omega\right)
\end{aligned}
$$


where $P(m=0)$, is the projection onto the states with zero mass, viz. the vacuum $\Omega$ and the one-particle states $\mathscr{H}_{1}$. An application of the techniques of the Jost-Lehmann-Dyson representation to the commutator function $x \rightarrow\left(\Omega,\left[A_{1}(x), A_{2}\right] \Omega\right) \quad$ as in Ref. [9] or [10] shows that the above expression vanishes if $A_{1}$ and $A_{2}$ are localized in spacelike separated double cones. For timelike separations of the operators we can exploit the support properties of solutions of the wave-equation or, more directly, part c) of Lemma 1:

$\left(\Omega, A_{1} P_{1} A_{2} \Omega\right)=\left(\Omega, A_{1} A_{2}^{\text {out }} \Omega\right)=\left(\Omega, A_{2}^{\text {out }} A_{1} \Omega\right)=\left(\Omega, A_{2} P_{1} A_{1} \Omega\right)$.

c) This statement follows from part c) of Lemma 1 and the selfadjointness of $\mathrm{A}_{1}^{\text {out }}$.

We conclude this chapter with the construction of the asymptotic field algebra $\mathfrak{F}^{\text {out }}$ and a brief discussion of its properties. Similarly as in Ref.[1] we define first of all local algebras $\mathfrak{F}^{\text {out }}\left(\boldsymbol{O}_{1}\right)$ attached to double cones $\boldsymbol{O}_{1}$ of arbitrary size and location:

$$
\mathcal{F}^{\text {out }}\left(O_{1}\right)=\left\{A^{\text {out }}: A=A^{*} \in \mathcal{F}\left(O_{1}\right) \cap \mathcal{F}_{N_{0}}\right\}^{\prime \prime},
$$

or in letters: $\mathcal{F}^{\text {out }}\left(\boldsymbol{O}_{1}\right)$ is the von Neumann algebra which is generated by all selfadjoint asymptotic field operators $A^{\text {out }}$ constructed from local operators $A \in \mathcal{F}\left(\boldsymbol{O}_{1}\right)$. In this definition we have restricted out attention to double cones, because it is only for such regions that we know that the algebras $\mathcal{F}^{\text {out }}\left(\sigma_{1}\right)$ and $\mathcal{F}^{\text {out }}\left(\sigma_{2}\right)$ commute: $\mathscr{F}^{\text {out }}\left(\sigma_{1}\right) \subset \mathcal{F}^{\text {out }}\left(\sigma_{2}\right)^{\prime}$ if $\sigma_{1}$ and $\sigma_{2}$ are spacelike separated. (Compare part b) of Theorem 9). However, there is a canonical way to extend the definiton of $\mathfrak{F}^{\text {out }}(6)$ to arbitraxy bounded regions $\mathcal{O}$ without loss of the commutation properties at spacelike distances: for a general region $\mathcal{O}$ we define $\mathcal{F}^{\text {out }}(6)$ as the von Neumann algebra which is generated by all $\mathfrak{F}^{\text {out }}\left(G_{1}\right)$ with $\sigma_{1} \subset \mathcal{O}$; the asymptotic field algebra $\mathfrak{F}^{\text {out }}$ is then the global $C^{*}$-algebra of all local algebras $\mathfrak{F}^{\text {out }}(6)$. It is obvious from the results in the present chapter that with our definition the net $\sigma \rightarrow \mathcal{F}^{\text {out }}(6)$ enjoys all the properties usually required in quantum field theory; it is in particular local and covariant.

As in the Fermi case [1] there are some geometrical relations between the net of the underlying field algebra $\mathfrak{F}$ and the net of the asymptotic algebra $\mathfrak{F}^{\text {aut }}$ : if $\mathcal{O}$ is any region, bounded or unbounded, with a non-trivial future tangent 
$\mathcal{O}_{+}$, then the asymptotic fields localized in $\mathcal{O}$ commute with the basic fields localized in $\boldsymbol{O}_{+}$,

$$
\mathcal{F}^{\text {out }}(6) \subset \mathcal{F}\left(G_{+}\right)^{\prime}
$$

(If 6 is an unbounded region we define $\mathcal{F}^{\text {out }}(6)$ as the smallest von Neumann algebra containing all algebras $\mathfrak{F}^{\text {out }}\left(\boldsymbol{O}_{1}\right)$ with $\boldsymbol{O}_{1} \subset \mathcal{O}$. .) This relation follows easily from part c) of Theorem 9. It is characteristic for massless particles and may be interpreted as the field-theoretic version of the Huyghens principle.

\section{Concluding Remarks}

It is a remarkable fact that the asymptotic field algebras do exist in all charge sectors which can be obtained from the vacuum with the aid of local fields or (more generally) localized morphisms [10]. The details of the model, in particular the superselection structure and the massive part of the particle spectrum are irrelevant for the construction. It seems therefore to be reasonable to base an analysis of the infra-particle problem (mentioned in the introduction) on these algebras. Although we do not hope for a complete solution within our general setting, we are optimistic that an analysis of the asymptotic algebras will yield at least a profound survey of the mathematical structures which are relevant to the description of collision processes of infra-particles.

In models with no infrared difficulties, e.g. if the massive particles have a precise mass and the collision states can be constructed a la Haag-Rue11e [11], all representations of $\mathfrak{F}^{\text {out }}$ induced by vectors $\Psi \in \mathcal{H}$ are equivalent to the vacuum representation. This means that one can specify for each vector $\Psi \in \mathcal{H}$

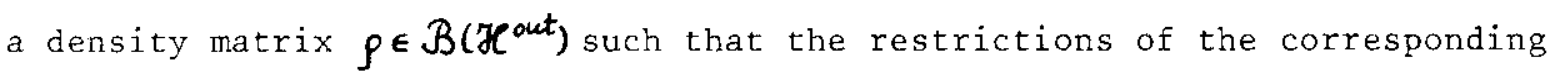
states to the algebra $\mathfrak{F}$ out coincide:

$$
(\Psi, C \Psi)=\operatorname{Tr} \rho C, C \in \mathcal{F}^{\text {out }}
$$

Hence the results of asymptotic field-strength measurements can always be interpreted in terms of asymptotic configurations of massless particles. It is another simple consequence of relation (23) that the weak closure of $\mathfrak{F}^{\text {out }}$ is isomorphic to the algebra of all bounded operators on $\mathcal{H}^{\text {out }}, \mathcal{F}^{\text {out }} \| \simeq \mathcal{B}^{\prime}\left(\mathcal{K}^{\text {out }}\right)$. Thus $\mathcal{F}^{\text {out }} /$ is a factor of type I (in terms of the classification scheme of von Neumann algebras [12]) and this feature seems to be typical for a situation with no infrared problems. 
If infinitely many, massless particles are produced in collisions, relation (23) does no longer hold and there appear other representations of $\mathcal{F}$ out besides the Fock-representation. This will manifest itself in a somewhat different

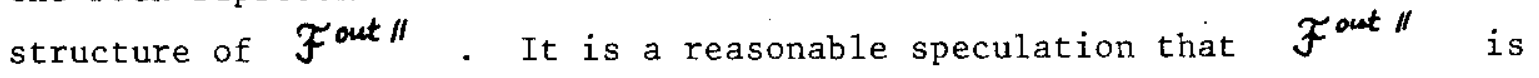
still type I in such a situation, however it should be no longer a factor. So we expect that $\mathcal{F}^{\text {out }}$ is in general isomorphic to $\beta$ ( $\left.\mathcal{L}^{\text {out }}\right) \bar{\otimes} \boldsymbol{\xi}$ where $z$ is the center of $\mathcal{F}$ out $\boldsymbol{\|}$. The inequivalent representations of $\mathfrak{F}$ out could then be distinguished by the elements of $z$ and the inevitable next question is: what is the physical significance of the elements of $z$ ? In order to give an idea of a possible answer we quote a remark of Fröhlich. He gives in a very interesting article [13] an argument that in models like quantum electrodynamics $z$ should be the algebra of the momenta of the charged particles. This result is in accord with the folk-1ore that different momentum distributions of charged particles give rise to inequivalent representations of the asymptotic photon algebras. One might hope that $\boldsymbol{z}$ admits such a simple phyiscal interpretation also in general.

Besides an anlysis of $\boldsymbol{z}$, which could be useful for a classification of the representations of $\mathcal{F}^{\text {out }}$ and an understanding of their global structure, it would be desirable to extract from the basic postulates some informations about the intrinsic properties of these representations. We believe that relation (22) (the field theoretical version of the Huyghens principle) could be an important tool in such investigations. For example, it follows quite easily from this relation that the, vectors $\Psi \in \mathcal{H}$ induce representations of the local algebras $\mathfrak{F}^{\text {out }}(\boldsymbol{O})$ ( $\mathcal{O}$ being any region with a non-trivial future tangent $G_{+}$) which are equivalent to the vacuum representation. So relation (23) holds also in general if one restrictsthe operators $C$ to the algebras $\mathcal{F}^{\text {out }}(\boldsymbol{0})$. The density matrix $\rho$ however will depend on the size of 0 and there exists in general no global $\rho$. For the proof of this assertion we use a fundamental result in the theory of von Neumann algebras [12, Theorem 2.7.9]: if a von Neumann algebra $\mathscr{H}$ on a Hilbert space $\mathscr{H}$ has a separating vector $\xi$, then every normal state $\boldsymbol{\psi}$ of $\mathcal{M}$ can be represented by a vector $\eta$ in the strong closure of the subspace $\{M \xi: M \in M\}$. In our example $\boldsymbol{M}$ is the algebra $\mathcal{F}^{\text {out }}(\sigma)$ and $\xi$ is the vacuum $\Omega$, which is cyclic for $\mathcal{F}\left(\sigma_{+}\right)$ and therefore (by relation $(22)$ ) separating for $\mathfrak{F}^{\text {out }}(0)$. Now every vector $\Psi \in \mathcal{H}$ gives rise to a normal state of $\mathcal{F}^{\circ}(0)$ and, according to the proposition quoted above, there exists a vector $\eta \in\left\{F \Omega: F \in \mathcal{F}^{\text {out }}\left(\boldsymbol{O}^{\prime}\right)\right\}^{(-)}=\mathcal{H}^{\text {out }}$ such that $(\Psi, C \Psi)=(\eta, C \eta)$ for all $C \in \mathcal{F}^{\text {out }}(6)$. This proves the statement. 
It is amazing that the frequently discussed coherent infrared representations (see e.g. [14]) are equivalent to the vacuum representation only for bounded regions [15]. In an evident contrast to oux result, this equivalence gets lost if 6 is an infinitely extended region, like the backward lightcone. So it seems as if the representations of $\mathcal{F}^{\circ}(0)$ which appear naturally in a field theoretical framework do have nicer properties than the coherent infrared representations, to which one is led by a study of models with external currents [16] or by a perturbative approach to field theory [17]. An explanation of this discrepancy could be an important step towards a solution of the infrared puzzle.

Another type of questions, worth while to look at within the context of the present studies, is related to the problem of spontaneous symmetry breaking. No systematic analysis of the observable consequences of a spontaneously broken symmetry has been carried out so far in the general framework of quantum field theory. There exist only some isolated results like the Goldstone theorem [ 4], which assures the existence of massless particles, or Adler's theorem on zeros of the S-matrix, which holds in certain models [18]. (For a review of the present status of the discussion see the recent article of Joos and Weimar [19]).

The alternatives in the field theoretical description of models with a spontaneously broken symmetry group $y$ are well known: either one insist on the irreducibility of the basic field algebra $\mathfrak{F}^{5}$, then $y$ cannot be unitarily implemented in $\mathscr{H}$ and acts only via automorphisms on $\mathcal{F}$. Or one uses a formulation in which $\mathscr{G}$ is unitarily implemented; then $\mathcal{F}$ is reducible and the vacuum $\Omega$ is not unique. Joos and Weimar advertise in their paper the second approach because it allows the application of group theoretical methods generally used in physics. We want to stress here that the apparent drawback of the second approach, the presence of many vacua, introduces no difficulties as far as the formulation of a collision theory for the massless particles is concerned. Going through our whole construction once more it is evident that the uniqueness of the vacuum is not crucial and can be given up. In models with a degenerate vacuum the main modifications are in Lemma 2 . Whereas the first part of this proposition remains unchanged the second half has to be replaced by

$$
\lim _{r \rightarrow \pm \infty}\left(\Omega, A_{1 T} \cdots A_{n \Gamma} \Omega\right)=\sum \int d \mu(\xi)\left(\Omega_{\xi}, A_{i_{1}} P_{1} A_{i_{2}} \Omega_{\xi}\right) \ldots\left(\Omega_{\xi}, A_{i_{n-1}} P_{1} A_{i_{n}} \Omega_{\xi}\right)
$$


where $d \mu(\xi)$ is a positive measure on the spectrum space of the center of $\mathcal{F}$ and $\xi \rightarrow \Omega_{\xi} \quad$ is the corresponding decomposition of the vacuum space $H_{0}$. (Of course the scalar products of the collision states have to be modified in a similar manner). So also in models with a degenerate vacuum a collision theory for the massless particles exists and seems to be an appropriate starting point for a systematic analysis of the observable consequences of spontaneously broken symutries.

\section{Appendix}

In this appendix we are concerned with the proof of Lemma 2 . This proposition is, in the massless case, a substitute for the well known bounds on vacuum expectation values of local operators at spacelike distances in theories with mass gap [6]. Fortunately, many of the basic ideas of Araki, Hepp and Ruelle expounded in Ref.[6] can be carried over also to the present case. So, properly speaking, our argument is nothing more but a rather tedious yet straightforwarà application of methods already developed.

To begin with we give a summary of the main steps in our proof. For this purpose let us have a look at the vacuum expectation values of the operators $A_{t}=t \cdot \int d \omega B(t, \underline{\underline{e}}) \quad, \quad B=-2 \partial_{0} A \quad$ defined in relation $(3):$
$\quad\left(\Omega, A_{1 t_{1}} \cdots A_{n t_{n}} \Omega\right)=t_{1} \cdots t_{n} \int d \omega_{1} \cdots \int d \omega_{n}\left(\Omega, B_{1}\left(t_{1}, t_{1} \underline{e}_{1}\right) \cdots B_{n}\left(t_{n}, t_{n} \underline{e}_{n}\right) \Omega\right)$.

Bearing in mind that the operators $A_{T}$ defined in relation (6) are averages of the operators $A_{t}$ at time $T$ over an interval of size $\ln |T|$, it is evident that our main task consists in verifying that the above expression is uniformly bounded in $t_{1}, \ldots t_{n}$ if all time difference $\left|t_{i}-t_{j}\right|$ are small compared to $t_{i}, t_{j}$. We shall do this by first converting the vacuum expectation value $\left(\Omega, B_{1} \cdots B_{n} \Omega\right)$ (where we have suppressed the coordinates for a moment) into a sum of vacuum expectation values of commutators. This is possible because of the spectrum condition which enables us to replace each operator $B_{i}$ acting on the vacuum by a creation operator $B_{i}^{+}$such that

$$
B_{i}^{+} \Omega=B_{i} \Omega \text { and }\left(B_{i}^{+}\right)^{*} \Omega=0 \text {. }
$$

So if we replace in $\left(\Omega, B_{1} \cdots B_{n} \Omega\right)$ the operator $B_{n}$ by $B_{n}^{+}$and commute it to the left, we get a sum of expressions each of which contains a 
commutator $\left[B_{i}, B_{n}^{+}\right]$. The remaining term with $B_{n}^{+}$on the extreme left vanishes owing to $\left(B_{n}^{+}\right)^{*} \Omega=0$. Now in some of the terms of this sum the commutators $\left[B_{i}, B_{n}^{+}\right]$are placed on the right of operators $B_{K}, B_{l}$. In these expressions we commute the commutators $\left[\mathbf{B}_{i}, \mathbf{B}_{n}^{+}\right]$to the left until they are placed next to the vacuum. Again we get contributions in which now double comnutators $\left[B_{i},\left[B_{j}, B_{n}^{+}\right]\right]$are placed on the right of operators $B_{k}, B_{\imath}$ and again we commute these double commutators to the left. We continue this procedure until all commutators are placed on the left, next to the vacuum, and all single operators $B_{k}, B_{l}$ are placed on the right of the commutators. Then we repeat the whole procedure: we replace the operator $B_{m}$ say, which is now next to the vacuum by $B_{m}^{+}$and commute it to the left. Then we commute all commutators originating from this procedure to the left until again all single operators $B_{k}, B_{l}$ are placed on the right of all commutators. Continuing this it is clear that we will finally arrive at a sum of vacuun expectation values containing only commutators. We give the first few expressions:

$$
\begin{aligned}
& n=2: \quad\left(\Omega, B_{1} B_{2} \Omega\right)=\left(\Omega,\left[B_{1}, B_{2}^{+}\right] \Omega\right) \\
& n=3: \quad\left(\Omega, B_{1} B_{2} B_{3} \Omega\right)= \\
&=\left(\Omega,\left[B_{1},\left[B_{2}, B_{3}^{+}\right]\right] \Omega\right)+\left(\Omega,\left[\left[B_{2}, B_{3}^{+}\right], B_{1}^{+}\right] \Omega\right)+\left(\Omega,\left[\left[B_{1}, B_{3}^{+}\right], B_{2}^{+}\right] \Omega\right) \\
& n=4: \quad\left(\Omega, B_{1} B_{2} B_{3} B_{4} \Omega\right)= \\
&=\left(\Omega,\left[B_{1}, B_{4}^{+}\right]\left[B_{2}, B_{3}^{+}\right] \Omega\right)+\left(\Omega,\left[B_{2}, B_{4}^{+}\right] \cdot\left[B_{1}, B_{3}^{+}\right] \Omega\right)+\left(\Omega,\left[B_{3}, B_{4}^{+}\right] \cdot\left[B_{1}, B_{2}^{+}\right] \Omega\right)+ \\
&+\left(\Omega,\left[B_{1},\left[B_{2},\left[B_{3}, B_{4}^{+}\right]\right]\right] \Omega\right)+\left(\Omega,\left[\left[B_{2},\left[B_{3}, B_{4}^{+}\right]\right], B_{1}^{+}\right] \Omega\right)+ \\
&+\left(\Omega,\left[\left[B_{1},\left[B_{3}, B_{4}^{+}\right]\right], B_{2}^{+}\right] \Omega\right)+\left(\Omega,\left[\left[\left[B_{3}, B_{4}^{+}\right], B_{2}^{+}\right], B_{1}^{+}\right] \Omega\right)+ \\
&+\left(\Omega,\left[\left[B_{1},\left[B_{2}, B_{4}^{+}\right]\right], B_{3}^{+}\right] \Omega\right)+\left(\Omega,\left[\left[\left[B_{2}, B_{4}^{+}\right], B_{3}^{+}\right], B_{1}^{+}\right] \Omega\right)+ \\
&+\left(\Omega,\left[\left[\left[B_{1}, B_{4}^{+}\right], B_{3}^{+}\right], B_{2}^{+}\right] \Omega\right) .
\end{aligned}
$$


Our reason for replacing simple quantities by a whole bunch of complicated looking expressions is of course that the consequences of locality are more transparent in the latter form. However, the whole procedure would be completely useless if we would have no control on the localisation properties of the operators $B^{+}$. We shall see that if $B$ is an element of one of the linear spaces $\mathcal{F}_{N}, B^{+}$can be chosen in such a way that it is quasi-local (in a sense which will be made precise later). Using locality we are then able to derive a uniform estimate for the spherical averages of multiple commutators, $m \geq 3$

$$
t_{1} \cdots t_{m} \cdot \int d \omega_{1} \cdots \int d \omega_{m}\left[B_{1}^{*}\left(t_{1}, t_{1} \underline{e}_{1}\right),\left[B_{2}^{*}\left(t_{2}, t_{2} \underline{e}_{2}\right),\left[\cdots, B_{m}^{*}\left(t_{m}, t_{m} \underline{e}_{m}\right)\right] \cdots\right]\right.
$$

where $B^{*}$ stands for $B$ or $B^{+}$. It turns out that the norms of these expressions decrease in the limit of large $t_{1} \approx t_{2} \approx \cdots \approx t_{m}$ like an inverse power of $t_{1}$, provided all operators $B_{1}, \ldots B_{m}$ are elements of $\mathfrak{F}_{N}$ for some sufficiently large $N$. For the commutator, $m=2$, we get an upper bound of the norm which is increasing in $t_{1}$. However, this increase is small compared to the decrease of the multiple commutators and this fact will allow us to neglect in the above vacuum expectation values a1l contributions which contain at least one multiple commutator. If $\mathfrak{n}$ is even we are then left with a sum of vacuum expectation values which contain only products of simple commutators. (Compare the above expressions for $n=2,3$ and 4). For the treatment of these expressions we have to analyze vectors like

$$
\left(1-P_{0}\right) \cdot t_{i} t_{j} \int d \omega_{i} \int d \omega_{i}\left[B_{i}\left(t_{i}, t_{i} e_{i}\right), B_{j}^{+}\left(t_{j}, t_{j} \underline{e}_{j}\right)\right] \Omega
$$

where $P_{0}$ is the projection onto the vacuum. Using locality and a bound on the 2-point function given in Ref.[6] we shall verify that the norms of these vectors decrease for large $_{i} \approx t_{j}$ fast enough to suppress a11 contributions coming from the remaining commutators. We may therefore insert in

$$
t_{1} \cdots t_{n} \cdot \int d \omega_{1} \cdots \int d \omega_{n}\left(\Omega,\left[B_{1}\left(t_{1}, t_{1} \underline{e}_{1}\right), B_{2}^{+}\left(t_{2}, t_{2} e_{2}\right)\right] \cdots\left[B_{n-1}\left(t_{n-1}, t_{n-1} \underline{e}_{n-1}\right), B_{n}\left(t_{n}, t_{n} \underline{e}_{n}\right)\right] \Omega\right)
$$

the projection $P_{0}$ between all commutators, the difference being negligible in the limit of large $t_{1} \approx t_{2} \approx \cdots \approx t_{n}$. It is then simple to infer from the resulting expression (which is a product of vacuum expectation values containing only one commutator) the assertion of the lemra.

After these qualitative remarks we come now to the quantitative statements. For the sake of clarity we split the text into four parts. 
a) The Creation Operators $\mathrm{B}^{+}$

Since the vacuum $\Omega$ is separating for all local operators it is obvious that the creation operators $\mathrm{B}^{+}$defined above cannot be strictly local. In order to have a measure which indicates how much they deviate from local operators we use the notion of quasi-localisation introduced by Araki and Haag [20]. We recall their definition briefly: let $\varphi_{R}$ be the double cone in configuration space which is the intersection of the forward cone with tip in $(-R, \underline{O})$ and the backward cone with tip in $(R, Q)$. Then an operator $F \in \mathfrak{F}$ is said to be quasi local of order $N$ if there exists for each (sufficiently large) $R>0$ an approximating local operator $F_{R} \in \mathcal{F}\left(\ell_{R}\right)$ such that

$$
\left\|F-F_{k}\right\| \leq c \cdot R^{-N}
$$

for some constant $c$. We shall demonstrate now that to each operator $E \in \mathbb{G}_{N}$

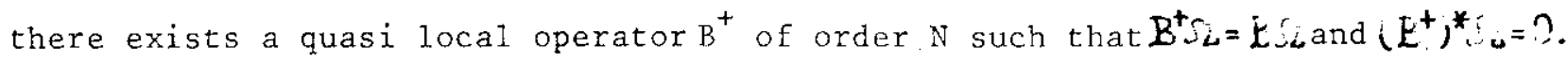

The elements of $\tilde{F}_{N}, N \in \mathbb{N}$. are finite sums of local operators $B$ of the form

$$
E=i\left(t+(t) i^{i t\left(t_{2}\right)} A e^{-i t\left(f_{2}\right)}\right.
$$

where $H=\widetilde{Z}_{n}$ and $f(t)$ is a testfunction with compact support which has a Fourier transform $\breve{f}(\omega)$ with an $N$-fold zero at $\omega=0$. If we apply such a $B$ to the vacuum we get, owing to the invariance of $\Omega$ under transtations,

$$
E \Omega=j d t f(t) e^{\left.i t(P)_{2}\right)} 4 .=j(\nu) E(d \omega) 4 . L
$$

where $E(d$,$\left.) is the spectral measure corresponding to the operator ( F_{\eta_{2}}\right)$. Now $\left(H_{2}\right)$ is a positive operator because of the spectrum condition and the fact that $n$ is a positive timelike vector. Therefore only positive values of $\omega$ contribute to the above $\omega$-integral and we may therefore replace in the $t$-integral the function $f(t)$ by $t^{+}(t)$, where

$$
f^{+}(t)=(2 \pi)^{-1} \int_{0}^{\infty} d \omega \tilde{F}(s) \iota^{-i t} .
$$

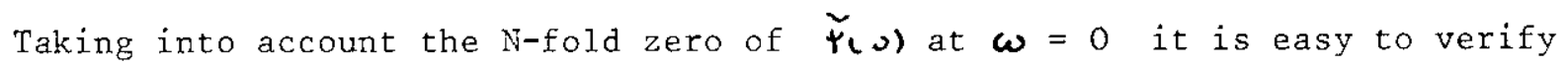
that $t^{+}(t)$ is continuous and $\quad\left|t^{+}(t)\right| \leq c \cdot|t|^{-N-1}$. Consequently the integral

$$
E^{+}=J d t+(t) v^{i t\left(P_{n}\right)} A e^{-i t\left(P_{n}\right)}
$$

exists as a Bochner integral. It follows also from the decrease properties of $\varphi^{+}(t)$ and the strict locality of $A$ that $B^{+}$is quasi local of order $\mathrm{N}$; 
we take as local approximations of $B^{+}$the operators $B_{R}^{+}, R \geq R_{0}$

$$
B_{R}^{+}=\int_{-a(R)}^{a(R)} d t f^{+}(t) e^{\left.i t i P_{n}\right)} A e^{-i t\left(P_{n}\right)} \text { with } \quad a(R)=\frac{k-K_{0}}{\sqrt{2} \cdot \ln \mid}
$$

where $R_{0}$ is the radius of the localisation region $\varphi_{R_{0}}$ of $A$ and $|n|$ is the Euclidean length of $n$. It will be important for our argument that the operators $B_{R}^{+}$are elements of $\mathfrak{F}_{C}$ and that $\left\|B_{R}^{+}\right\| \leq c,\left\|\partial_{0} B_{R}^{+}\right\| \leq c^{\prime}$ uniformly in $R$.

The support properties of $\varphi^{+}$in momentum space imply $B^{+} \Omega=E \Omega$ and $\left(\mathrm{E}^{+}\right)^{*} \Omega=0$ So $\mathrm{B}^{+}$is an operator with the desired properties. Since the elements of $\widetilde{F}_{N}$ are linear combinations of operators similar to $B$ the above construction can be performed just so for any operator from $\tilde{Z}_{N}$.

b) Bounds on Multiple Comnutators

We shall estimate now multiple commutators of the operators

$$
A_{t}^{*}=t \cdot \int d \omega E^{*}(t, t \underline{t})
$$

and their time averages. The proofs are based only on locality.

Lemma:

a) Let $B_{1}, \ldots B_{n}$ be operators which are localized in double cones

$\varphi_{R_{1}}, \ldots \varepsilon_{k_{n}}$. If all $t_{i} i=1, \ldots, n$ are positive (or negative resp.) then

$$
\begin{aligned}
& \int d \omega_{1} \cdots \int d \nu_{n} \|\left[B_{1}\left(t_{1}, t_{1} \epsilon_{1}\right),\left[B_{2}\left(t_{2}, t_{2} e_{2}\right),\left[\cdots, B_{n}\left(t_{n}, t_{n} \epsilon_{n}\right)\right] \cdots j \|\right.\right. \\
& \leq\left\|E_{1}\right\| \cdots\left\|b_{n}\right\| \cdot \prod_{k=1}^{n-1}\left(\sum_{l=k+1}^{n} \frac{R_{k l}^{2}+2 R_{k l} \cdot\left|t_{k}-t_{2}\right|}{2 t_{k} t_{l}}\right) \\
& \text { where } R_{k l}=R_{k}+k_{l} .
\end{aligned}
$$

b) If $B_{1}, \ldots B_{n}$ are quasi local of order $N$ then for any $R>0$

$$
\begin{aligned}
& \int d \omega_{1} \cdots \int d \omega_{n} \|\left[E_{1}\left(t_{1}, t_{1} \varepsilon_{1}\right), L B_{2}\left(t_{2}, t_{2} E_{2}\right),\left[\cdots, E_{n}\left(t_{n}, t_{n} \underline{e}_{n}\right)\right] \cdots j \|\right. \\
& \leq-\sum_{i} \frac{1}{k^{N}}+\prod_{k=1}^{n-1}\left(\sum_{i=k+1}^{n} \frac{R^{2}+2 k \cdot i t_{k}-t_{i} i}{2 t_{k} t_{l}}\right)
\end{aligned}
$$

and the number $c$ does not depend on $R$ and $t_{1}, \ldots t_{n}$. 


\section{Proof:}

a) We prove the first half of the lemma by induction. For $n=2$ we have to consider

$$
\int a \nu_{1} \int d \nu_{2}\left\|L E_{1}\left(t_{1}, t_{1} t_{1}\right), L_{2}\left(t_{2}, t_{2} t_{2}\right) d\right\|
$$

Owing to locality the integrand of this expression vanishes for all $\epsilon_{1}, \underline{\varepsilon}$ for which the two inequalities $\left|t_{1}-t_{2} \pm k_{12}\right|^{2} \leq\left|t_{1} \leqslant_{1}-t_{2} \leqslant_{2}\right|^{2}$ hold. Consequently we have to integrate only over the region $r_{12}-u^{2} \times u^{2}:$

$$
\tau_{12}=\left(t_{1}\right) t_{2}: n \leq 1-\left(t_{i} t_{2}\right) \leq \frac{k_{12}^{2}+\alpha k_{12} \cdot\left|t_{1}-t_{2}\right|}{2 t_{1} t_{2}} \text {;. }
$$

Thus, if $\chi_{12}$ denotes the characteristic function of $G_{12}$, we can estimate $j a \nu_{1} j u \nu_{2}\left\|\left(t_{1}\left(t_{1}, t_{1} t_{1}\right), t_{2}\left(t_{2}, t_{2} t_{2}\right)\right\rfloor\right\|$

$\leq \alpha \cdot\left\|b_{1}\right\|\left\|c_{2}\right\| \cdot d \nu_{1 j} u \nu_{2} l_{12}\left(t_{1}, \underline{k}_{2}\right) \leq\left\|t_{1}\right\|\left\|t_{2}\right\| \cdot \begin{gathered}K_{12}^{2}+\alpha K_{12} \cdot\left|t_{1}-t_{2}\right| \\ \alpha t_{1} t_{2}\end{gathered}$ and this proves the lemma for $n=2$. Let us assume now that the lemma holds for $\mathrm{n}-1$. Then we get, using locality once more,

$$
\begin{aligned}
& \left.\int d \omega_{1} \cdots J d \omega_{n} \| L B_{1}\left(t_{1}, t_{1} \underline{e}_{1}\right), L B_{2}\left(t_{2}, t_{2} \underline{t}_{2}\right), L \cdots, t_{n}\left(t_{n}, t_{n} \underline{t}_{n}\right)\right\rfloor \cdots, \|
\end{aligned}
$$

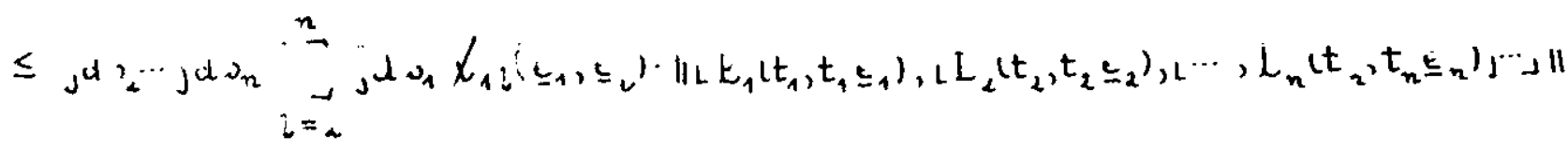

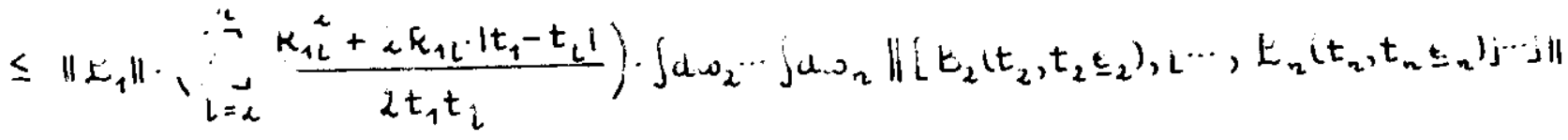

$$
\begin{aligned}
& =\left\|L_{1}\right\| \cdots\left\|E_{n}\right\| \cdot \prod_{k=1}^{n-1} \prod_{i=k+1}^{2} \frac{k_{k L}^{2}+2 k_{k L} \cdot\left|t_{k}-t_{L}\right|}{2 t_{k} t_{2}}
\end{aligned}
$$

where $\chi_{12}$ is the characteristic function of the region $\hat{T}_{1 l}$ which is defined in analogy to $G_{12}$.

b) Since the operators $B_{1}, \ldots, B_{n}$ are quasi local of order $N$ we can find approximating sequences $B_{1 R}, \ldots, B_{n R}$ which are localized in the double cone o such that

$$
\left\|E_{i}-E_{i R}\right\| \leq c_{i} \cdot k^{-N}, \quad i=1, \ldots n .
$$


Then we split every operator $B_{i}$ in the above expression into two parts, $B_{i}=B_{i R}+\left(B_{i}-B_{i R}\right), \quad$ obtaining a sum of $2^{n}$ terms. In one of these terms there appear only operators $B_{i R}$ and we can apply to it part a) of the lemma. Each term in the remaining sum contains at least one operator $\left(B_{i}-B_{i R}\right)$ and can therefore be estimated by $c \cdot R^{-N}$ with a suitable constant $c$.

We apply now the above lemma for an estimate of multiple commutators of the time averages

$$
A_{T}^{*}=\int d t h_{r}(t) A_{t}^{\#}
$$

where $h_{T}$ is the function defined in relation (5).

\section{Proposition I:}

Let $B_{1}, \ldots B_{n}$ be operators from $\mathscr{F}_{N}$. Then

$$
\|\left[A_{1 T}^{*},\left[A_{2 T}^{*},\left[\cdots, A_{n T}^{*}\right] \cdots\right] \| \leq c \cdot|T|^{-[N(n-2)-2 n(n-1)] /[N+2(n-1)]}\right.
$$

for large $|T|$. The constant $c$ does not depend on $T$.

\section{Remark:}

If $\mathrm{n}=2$, the bound given above increases with $|\mathrm{T}|$ like $|\mathrm{T}|^{4 /[\mathrm{N}+2]}$ However for $n \geq 3$ and $N$ sufficiently large, the bound decrease almost like $\mid T !^{-[n-2]}$.

\section{Proof:}

Since the operators $B_{1}, \ldots, B_{n}$ are elements of $\mathscr{F}_{N}$, the corresponding creation operators $B_{1}^{+}, \ldots, B_{n}^{+}$are quasilocal of order $N$. On the other hand every local operator is also quasilocal (of any order) and we can apply part b) of the preceding lemma to the operators $B_{1}^{*}, \ldots, B_{n}^{*}$ :

$$
\begin{aligned}
& \|\left[A_{1 T}^{*},\left[A_{2 \tau}^{*},\left[\cdots, A_{n}^{*}\right] \cdots\right] \|\right. \\
\leq & \int d t_{1} \cdots \int d t_{n}\left|h_{\tau}\left(t_{1}\right) \cdots h_{T}\left(t_{n}\right)\right| \cdot\left|t_{1} \cdots t_{n}\right| \cdot \int d \omega_{1} \cdots \int d \omega_{n}\left\|\left[B_{1}^{*}\left(t_{1}, t_{1} e_{1}\right),\left[\cdots, B_{n}^{*}\left(t_{n}, t_{n} \underline{e}_{n}\right)\right] \cdots\right]\right\| \\
\leq & \int d t_{1} \cdots \int d t_{n}\left|h_{T}\left(t_{1}\right) \cdots h_{r}\left(t_{n}\right)\right| \cdot\left|t_{1} \cdots t_{n}\right| \cdot c\left\{\frac{1}{R^{N}}+\prod_{k=1}^{n-1}\left(\sum_{l=k+1}^{n} \frac{R^{2}+2 R \cdot\left|t_{k}-t_{l}\right|}{2 t_{k} t_{l}}\right)\right\} .
\end{aligned}
$$

Taking into account the support properties of $h_{T}$ we get after integration 


$$
\|\left[A_{1}^{*},\left[A_{2}^{*},\left[\cdots, A_{n T}^{*}\right] \cdots\right] \| \leq c^{\prime} \cdot|T|^{n} \cdot\left\{\frac{1}{R^{N}}+\left(\frac{R^{2}+2 R \cdot \ln |T|}{|T|^{2}}\right)^{n-1}\right\}\right.
$$

for sufficiently large ITI with a constant $c$ ' which does not depend on $R$ and $|T|$. This inequality holds for arbitrary $R>0$ and if we put $R=|T|^{2(n-1) /[N+2(n-1)]}$ the statement follows.

\section{c) The Vacuum Expectation Value of Two Commutators}

Since our bound on the norm of the commutator $\left[A_{1 T}^{*}, A_{2 T}^{*}\right]$ is too weak for later applications we have still to refine our analysis. We shall estimate in the following the vacuum expectation value of two such commutators. In these investigations we shall benefit from a result of Araki, Hepp and Ruelle [6] on the two-point function:

\section{Lemima:}

Let $C_{1}$ and $C_{2}$ be two local operators from $\mathfrak{F}_{0}$ which are localized in $\sigma_{1}$ and $G_{2}$. Then for all $|\underline{x}| \geq 2\left(R_{1}+R_{2}\right)$

$$
\left|\left(\Omega, C_{1}(\underline{x}) \cdot\left(1-P_{0}\right) C_{2} \Omega\right)\right| \leq c \cdot \frac{\left(R_{1}+R_{2}\right)^{3}}{|\underline{x}|^{2}} \cdot\left\{\left\|C_{1}^{*} \Omega\right\| \cdot\left\|\partial_{0} C_{2} \Omega\right\|+\left\|C_{2}^{*} \Omega\right\| \cdot\left\|\partial_{0} C_{1} \Omega\right\|\right\}
$$

where $P_{0}$ is the projection onto the vacuum and $c$ is a constant which depends neither on $x$ nor on $C_{1}, C_{2}$ and $R_{1}, R_{2}$.

In the following we take for the operators $C_{i} i=1,2$ the commutators $\left[B_{j}\left(t_{j}, t_{j} \underline{e}_{j}\right), B_{k}\left(t_{k}, t_{k} \underline{e}_{k}\right)\right]$. It will be crucial for our argument that these operators are (for arbitrary $t_{j}$ and $t_{k}$ ) localized in regions with a finite volume, provided the time difference $\left|t_{j}-t_{k}\right|$ is kept small.

\section{Coro11ary:}

Let $B_{1}, \ldots, B_{4}$ be operators from $\mathfrak{F}_{0}$ which are localized in double cones $\varphi_{R_{1}}, \ldots \varphi_{R_{4}}$. Then for arbitrary positive (or negative resp.) $t_{1}, \ldots, t_{4}$ :

$\left|\left(\Omega,\left[A_{1 t_{1}}, A_{2 t_{2}}\right] \cdot\left(1-P_{0}\right)\left[A_{3 t_{3}}, A_{4 t_{4}}\right] \Omega\right)\right| \leq C \cdot \frac{R^{6}}{t^{2}} \cdot\left(1+\ln \left[1+\left(\frac{t}{R}\right)^{2}\right] \cdot R \sum_{i=1}^{4} \frac{\left\|d_{0} B_{i}\right\|}{\left\|B_{i}\right\|}\right) \cdot\left\|B_{1}\right\| \cdots\left\|B_{4}\right\|$ 
where $\quad t=\frac{1}{4}\left(t_{1}+t_{2}+t_{3}+t_{4}\right) \quad$ and

$$
R=\frac{1}{2}\left(1+|t| \cdot\left(t_{1} t_{2}\right)^{-1 / 2}+|t| \cdot\left(t_{3} t_{4}\right)^{-1 / 2}\right) \cdot \sum_{i=1}^{4}\left(R_{i}+\left|t-t_{i}\right|\right) .
$$

The constant $c$ depends neither on $t_{1}, \ldots, t_{4}$ nor on $B_{1}, \ldots, B_{4}$ and $R_{1}, \ldots, R_{4}$.

\section{Proof:}

We consider

$$
K=\left(\Omega,\left[B_{1}\left(t_{1}, t_{1} e_{1}\right), B_{2}\left(t_{2}, t_{2} e_{2}\right)\right] \cdot\left(1-P_{0}\right)\left[B_{3}\left(t_{3}, t_{3} e_{3}\right), B_{4}\left(t_{4}, t_{4} \underline{e}_{4}\right)\right] \Omega\right) .
$$

Because of the invariance of the vacuum under translations we can rewrite this expression according to

$$
K=\left(\Omega,\left[B_{1}\left(t-t, \underline{r}_{12}\right), B_{2}\left(t_{2}-t, \underline{r}_{21}\right)\right] u(\underline{x})^{-1}\left(1-P_{0}\right)\left[B_{3}\left(t_{3}-t, r_{34}\right), B_{4}\left(t_{4}-t, \underline{r}_{43}\right)\right] \Omega\right)
$$

where $\quad \underline{I}_{i k}=\frac{1}{2}\left(t_{i} e_{i}-t_{k} \underline{e}_{k}\right)$ and $\quad \underline{x}=\frac{1}{2}\left(t_{1} \underline{e}_{1}+t_{2} \underline{e}_{2}-t_{3} e_{3}-t_{4} \underline{e}_{4}\right)$.

Now the commutator $\left[B_{i}\left(t_{i}-t, r_{i k}\right), B_{k}\left(t_{k}-t, I_{k i}\right)\right] \quad$ is localized in the double cone $\varphi_{\boldsymbol{t}}$ with

$$
T=\left|\underline{r}_{i k}\right|+R_{i}+R_{k}+\left|t_{i}-t\right|+\left|t_{k}-t\right|
$$

On the other hand it is zero (owing to locality) if

$$
2\left|\underline{r}_{i k}\right| \geq R_{i}+R_{k}+\left|t_{i}-t\right|+\left|t_{k}-t\right| \text {. }
$$

Since the zero operator is localized in all regions we get for $r$ the bound

$$
r \leq \frac{3}{2}\left(R_{i}+R_{k}+\left|t_{i}-t\right|+\left|t_{k}-t\right|\right) \text {. }
$$

We can then apply the preceding lemma and get for $|x| \geq 3 \cdot \sum_{i=1}^{4}\left(R_{i}+\left|t_{i}-t\right|\right)$

$|K| \leq c \cdot \frac{1}{|\underline{x}|^{2}} \cdot\left(\sum_{i=1}^{4}\left(R_{i}+\left|t-t_{i}\right|\right)\right)^{3} x$

$\times\left\{\left\|\left[B_{1}\left(t_{1}, t_{1} \underline{e}_{1}\right), B_{2}\left(t_{2}, t_{2} \underline{e}_{2}\right)\right]^{*} \Omega\right\| \cdot\left\|\left[\partial_{0} B_{3}\left(t_{3}, t_{3} e_{3}\right), B_{4}\left(t_{4}, t_{4} e_{4}\right)\right] \Omega+\left[B_{3}\left(t_{3}, t_{3} \underline{e}_{3}\right), \partial_{0} B_{4}\left(t_{4}, t_{4} \underline{e}_{4}\right)\right] \Omega\right\|\right.$

$+(1 \longleftrightarrow 3,2 \longleftrightarrow 4)\}$. 
Now the commutators in this expression vanish if

$$
\left|\underline{e}_{1}-\underline{e}_{2}\right|^{2} \geq \frac{\left(R_{1}+R_{2}\right)^{2}+2\left(R_{1}+R_{2}\right) \cdot\left|t_{1}-t_{2}\right|}{2 t_{1} t_{2}}
$$

and analogously for $\left|\underline{e}_{3}-\underline{e}_{4}\right|^{2}$. We may therefore estimate $|\underline{x}|$ in the expression $(*)$ by

$$
\begin{aligned}
|\underline{x}| & =\frac{1}{2}\left|t_{1} \underline{e}_{1}+t_{2} \underline{e}_{2}-t_{3} \underline{e}_{3}-t_{4} \underline{e}_{4}\right| \geq|t| \cdot\left(\left|\underline{e}_{2}-\underline{e}_{3}\right|-\frac{1}{2}\left|\underline{e}_{1}-\underline{e}_{2}\right|-\frac{1}{2}\left|\underline{e}_{3}-\underline{e}_{4}\right|\right)-\frac{1}{2} \sum_{i=1}^{4}\left|t_{i}-t\right| \\
& \geq|t| \cdot\left|\underline{e}_{2}-\underline{e}_{3}\right|-\frac{1}{2}|t| \cdot\left(\frac{R_{1}+R_{2}+\left|t_{1}-t_{2}\right|}{\left(t_{1} t_{2}\right)^{1 / 2}}+\frac{R_{3}+R_{4}+\left|t_{3}-t_{4}\right|}{\left(t_{3} t_{4}\right)^{1 / 2}}\right)-\frac{1}{2} \sum_{i=1}^{4}\left|t_{i}-t\right| \\
& \geq|t| \cdot\left|\underline{e}_{2}-\underline{e}_{3}\right|-R
\end{aligned}
$$

where $R$ is the quantity defined in the formulation of the proposition. It is then straightforward to verify that for $|t| \cdot\left|\underline{e}_{2}-\underline{e}_{3}\right| \geq 4 R$

$$
|K| \leq c \cdot \frac{R^{3}}{t^{2} \cdot\left|\underline{e}_{2}-\underline{e}_{3}\right|^{2}+R^{2}} \cdot\{\ldots\} .
$$

The curly bracket coincides with that given in relation $(*)$. For $|t| \cdot\left|\underline{e}_{2}-\underline{e}_{3}\right| \leq 4 R$ we estimate

$$
|K| \leq\left\|\left[B_{1}\left(t_{1}, t_{1} e_{1}\right), B_{2}\left(t_{2}, t_{2} e_{2}\right)\right]^{*} \Omega\right\| \cdot\left\|\left[B_{3}\left(t_{3}, t_{3} e_{3}\right), B_{4}\left(t_{4}, t_{4} e_{4}\right)\right] \Omega\right\|+(1 \leftrightarrow 3,2 \leftrightarrow 4) .
$$

If we perform now the spherical integrations and use the bounds on the norms of the commutators given in the preceding section we get

$$
\begin{aligned}
& \int d \omega_{1} \cdots \int d \omega_{4}|K| \\
\leq & \left(c_{1} \cdot \int_{0}^{2} d \zeta \frac{R^{3}}{t^{2} \zeta+R^{2}} \cdot \sum_{i=1}^{4} \frac{\left\|\partial_{0} B_{i}\right\|}{\left\|B_{i}\right\|}+c_{2} \cdot \int_{0}^{8 \cdot(R / t)^{2}} d \zeta\right) \cdot \frac{\left(R_{1}+R_{2}+\mid t_{1}-t_{2} \|\right)^{2} \cdot\left(R_{3}+R_{4}+\mid t_{3}-t_{4}\right)^{2}}{t_{1} t_{2} t_{3} t_{4}} \cdot\left\|B_{1}\right\| \cdots B_{4} \| \\
\leq & \frac{c}{t_{1} t_{2} t_{3} t_{4}} \cdot \frac{R^{6}}{t^{2}} \cdot\left(\ln \left[1+\left(\frac{t}{R}\right)^{2}\right] \cdot R \sum_{i=1}^{4} \frac{\left\|\partial_{0} B_{i}\right\|}{\left\|B_{i}\right\|}+1\right) \cdot\left\|B_{1}\right\| \cdots B_{4} \|
\end{aligned}
$$

and this completes the proof.

Integrating the operators $A_{t}$ with the functions $h_{T}$ we arrive at the

Proposition II:

Let $B_{1}, \ldots, B_{4}$ be operators from $\mathfrak{F}_{N}$. Then for large $|T|$

$$
\left|\left(\Omega,\left[A_{1 T}^{*}, A_{2 T}^{*}\right] \cdot\left(1-P_{0}\right)\left[A_{3 T}^{*}, A_{4 T}^{*}\right] \Omega\right)\right| \leq c \cdot \ln |T| \cdot|T|^{-2(N-14) /(N+7)}
$$

where $c$ is a constant which does not depend on $T$. 


\section{Proof:}

If the operators $B_{1}, \ldots, B_{4} \in \mathcal{F}_{0}$ are localized in $\varphi_{R_{1}}, \ldots \varphi_{R_{4}}$ it follows from the support properties of $h_{\boldsymbol{r}}$ and the preceding corollary that for sufficiently large $|T|$ (depending on the function $h$ used in the definition of $h_{T}$ )

$$
\left|\left(\Omega,\left[A_{1 T}, A_{2 r}\right] \cdot\left(1-P_{0}\right)\left[A_{3}, A_{4 T}\right] \Omega\right)\right|
$$

$\leq c \cdot \frac{1}{T^{2}} \ln |T| \cdot\left[R_{0} \ln |T|+\sum_{i=1}^{4} R_{i}\right]^{6} \cdot\left[1+\left(R_{0} \cdot \ln |T|+\sum_{j=1}^{4} R_{j}\right) \cdot \sum_{k=1}^{4} \frac{\left\|\partial_{0} B_{k}\right\|}{\left\|B_{k}\right\|}\right] \cdot\left\|B_{1}\right\| \cdots\left\|B_{4}\right\|$.

Here the constants $c$ and $R_{0}$ depend only on $h$. Now if $B_{1}, \ldots, B_{4}$ are elements of $\mathcal{F}_{N}$ the operators $B_{1}^{*}, \ldots, B_{4}^{*}$ are quasilocal of order $N$. We may therefore split the operators $B_{i}^{*}$ in

$$
L=\left(\Omega,\left[A_{1 T}^{*}, A_{2 T}^{*}\right] \cdot\left(1-P_{0}\right)\left[A_{3 T}^{*}, A_{4 T}^{*}\right] \Omega\right)
$$

into the parts $B_{i}^{*}=B_{i R}^{*}+\left(B_{i}^{*}-B_{i R}^{*}\right)$ where $B_{i R}^{*}$ are the approximations of $B_{i}^{*}$ constructed in part a) of this appendix. To the term which contains exclusively operators $B_{i R}^{*}$ we apply the above inequality, bearing in mind that $B_{i R}^{*}$ is localized in $\varphi_{R}$ and $\left\|B_{i R}^{*}\right\| \leq c_{i},\left\|\partial_{0} B_{i R}^{*}\right\| \leq c_{i}^{\prime}$ uniformly in $R$. For the remainder we make use of $\left\|B_{i}^{*}-B_{i R}^{*}\right\| \leq C_{i}^{\prime \prime} \cdot R^{-N}$ getting altogether (for sufficiently large $|\mathrm{T}|$ ):

$$
|L| \leq c_{1} \cdot \frac{T^{4}}{R^{N}}+c_{2} \cdot \frac{1}{T^{2}} \ln |T| \cdot\left(R_{0} \ln |T|+R\right)^{7}
$$

and $c_{1}, c_{2}$ do not depend on $R$ and $T$ : If we set now $R=|T|^{6 /(N+7)}$ the statement follows.

\section{d) Proof of Lemma 2}

We are now furnished with the informations needed to complete the argument. First we consider the operator

$$
A_{t}=t \cdot \int d \omega B(t, t \underline{e}) \text { where } B=-2 \partial_{0} A .
$$

It follows easily from the definition of the space $\mathfrak{F}_{N}$ that with $A \in \mathcal{F}_{N}$ also $-2 \partial_{0} A \in \mathcal{F}_{N}$ and we may therefore disregard the special form of $B$ for a moment. Then we convert the vacuum expectation values of the time averages $A_{T},\left(\Omega, A_{1} \cdots A_{n T} \Omega\right)$, into a sum of terms containing only commutators $\left[A_{1}^{*},\left[A_{2 T}^{*},\left[\cdots, A_{m}^{*}\right] \cdots\right]\right.$. As was explained above there are two types of contributions. In the first one there is at least one multiple commutator with $m \geq 3$. To such a term we apply Proposition $I$ and get 
$\left|(\Omega, \underbrace{[\cdot,[\cdots] \cdots]}_{m_{1}} \cdots \underbrace{,[\cdots] \cdots]}_{m_{k}} \Omega)\right| \leq c \cdot \prod_{l=1}^{k}|T|^{-\left[N\left(m_{l}-2\right)-2 m_{2}\left(m_{2}-1\right)\right] /\left[N+2\left(m_{l}-1\right)\right]}$ in an obvious notation. Since $\sum_{l=1}^{k} m_{l}=n$, and since there exists by assumption at least one $m_{i} \geq 3$ we can estimate

$$
\sum_{l=1}^{K} \frac{N\left(m_{l}-2\right)-2 m_{l}\left(m_{l}-1\right)}{N+2\left(m_{l}-1\right)} \geq 1-\frac{6 n(n-1)}{N}
$$

This shows that these contributions converge to zero in the limit of large $|T|$ provided all operators $B_{1}, \ldots B_{n}$ are elements of $\mathfrak{F}_{N}$ with $N>6 n\left(r_{L}-1\right)$. If $\mathrm{n}$ is even the remaining contributions are of the form

$$
M_{n}(T)=\left(\Omega,\left[A_{1} T, A_{2 T}^{+}\right] \cdots\left[A_{n-1} T, A_{n T}^{+}\right] \Omega\right) .
$$

We shall prove by induction that if $N \geq 2 n+15$ then $M_{n}(T)$ converges in the limit of large ITI to a product of one-particle scalar products. For $n=2$ the statement follows from Lemma 1 , remembering that $B_{i}=-2 \partial_{0} A_{i}$ :

$$
\lim _{r \rightarrow \pm \infty} M_{2}(T)=\lim _{T \rightarrow \pm \infty}\left(\Omega,\left[A_{1} r, A_{2 T}^{+}\right] \Omega\right)=\lim _{T \rightarrow \pm \infty}\left(A_{1 T}^{*} \Omega, A_{2 T} \Omega\right)=\left(\Omega, A_{1} P_{1} A_{2} \Omega\right) .
$$

Let us assume now that the statement holds for $(n-2)$. If we replace in the above expression for $M_{n}(T)$ the unit operator 1 at the commutator on the left by the sum $\quad 1=P_{0}+\left(1-P_{0}\right)$ we get:

$$
\begin{aligned}
M_{n}(T) & =\left(\Omega,\left[A_{1 T}, A_{2 T}^{+}\right] \Omega\right)\left(S_{n},\left[A_{3 T}, A_{4 T}^{+}\right] \cdots\left[A_{n-1 T}, A_{n T}^{+}\right] \Omega\right) \\
& +\left(\Omega,\left[A_{1 T}, A_{2 T}^{+}\right] \cdot\left(1-P_{0}\right)\left[A_{3 T}, A_{4 T}^{+}\right] \cdots\left[A_{n-1 T}, A_{n T}^{+}\right] \Omega\right)
\end{aligned}
$$

The first expression converges by assumption to $\left(\Omega, A_{1} P_{1} A_{2} \Omega\right) \cdots\left(\Omega, A_{n-1} P_{1} A_{n} \Omega\right)$. The second term can be estimated, using Proposition $I$ and II, by

$$
\begin{aligned}
& \left|\left(\Omega,\left[A_{1 T}, A_{2 T}^{+}\right] \cdot\left(1-P_{0}\right)\left[A_{3 T}, A_{4 T}^{+}\right] \cdots\left[A_{n-1 T}, A_{n T}^{+}\right] \Omega\right)\right| \\
\leq & \left\|\left(1-P_{0}\right)\left[A_{1 T}, A_{2 T}\right]^{*} \Omega\right\| \cdot\left\|\left[A_{3 T}, A_{4 T}^{+}\right]\right\| \cdots\left\|\left[A_{n-1 T}, A_{n T}^{+}\right]\right\| \\
\leq & c \cdot(\ln |T|)^{1 / 2} \cdot|T|^{-(N-14) /(N+7)} \cdot|T|^{2(n-2) /(N+2)} .
\end{aligned}
$$


It is obvious that this expression approaches zero in the limit of large $|T|$ if $N \geq 2 n+15$ and therefore

$$
\lim _{T \rightarrow \pm \infty} M_{n}(T)=\left(\Omega, A_{1} P_{1} A_{2} \Omega\right) \cdots\left(\Omega, A_{n-1} P_{1} A_{n} \Omega\right)
$$

Summing up, we get for even $\mathrm{n}$ after some combinatorics

$$
\lim _{r \rightarrow \pm \infty}\left(\Omega, A_{1 T} \cdots A_{n T} \Omega\right)=\sum\left(\Omega, A_{i_{1}} P_{1} A_{i_{2}} \Omega\right) \cdots\left(\Omega, A_{i_{n-1}} P_{1} A_{i_{n}} \Omega\right)
$$

provided $A_{1}, \ldots A_{n} \in \mathbb{F}_{N}, N$ sufficiently large. The sum extends over all ordered pairs $i_{1}<i_{2}, \ldots i_{n-1}<i_{n}$ taken from $(1, \ldots n)$. For odd $n$ the limit vanishes, because in this case there do not appear terms of the type $M_{n}(T)$. This proves the second part of Lema 2. The first part is then an elementary consequence. 
[1] D. Buchholz: Collision Theory for Massless Fermions

Commun. math. Phys. 42, 269 (1975)

[2] D. Buchholz: Collision Theory for Waves and a Characterisation of Models with Trivial S-Matrix

Commin. math. Phys. 45, 1 (1975)

[3] B. Schroer: Infrateilchen in der Quantenfeldtheorie Fortschr. der Physik 11, 1 (1963)

[4] J. Goldstone, A. Salam, S. Weinberg:

Broken Symmetries

Phys. Rev. 127, 965 (1962)

H. Ezawa, J. A. Swieca:

Spontaneous Breakdown of Symmetries and Zero-Mass States Commun. math. Phys. $\underline{5}, 330$ (1967)

[5] R. Ferrari, L. E. Picasso:

Some Remarks on Local Operatos in Quantum Electrodynamics Commun. math. Phys. 35, 25 (1974)

[6] H. Araki, K. Hepp, D. Ruelle:

On the Asymptotic Behaviour of Wightman Functions in Space-Like Directions

Helv. Phys. Acta 35, 164 (1962)

K. Hepp: Proc. Brandeis University Summer Institute in Theoretical Physics 1965, Vol.1, p.137.

New York: Gordon and Breach 1966

[7] T. Kato: Perturbation Theory for Linear Operators Berlin-Heidelberg-New York: Springer 1966 
[8] M. Reed, B. Simon:

Methods of Modern Mathematical Physics II

(Fourier Analysis, Self-Adjointness)

New-York: Academic Press 1975

[9] L. J. Landau: Asymptotic Locality and the Structure of Local

Internal Symmetries

Commun. math. Phys. 17, 156 (1970)

[10] S. Doplicher, R. Haag, J. E. Roberts:

Local Observables and Particle Statistics II

Commun. math. Phys. 35, 49 (1974)

[11] R. Haag: Quantum Field Theories with Composite Particles and Asymptotic Behaviour

Phys. Rev. 112, 669 (1958)

D. Ruelle: On the Asymptotic Condition in Quantum Field Theory. Helv. Phys. Acta 35, 147 (1962)

[12] S. Sakai: C-algebras and $\mathrm{W}$-algebras Berlin-Heidelberg-New York: Springer 1971

[13] J. Fröhlich: The Asymptotic Condition and the Structure of Scattering States in Relativistic Quantum Mechanics with Massless One Particle States. Unpublished Notes (1974)

[14] G. Roepstorff:Coherent Photon States and Spectral Condition Comnun. math. Phys, 19, 301 (1970)

[15] A. Stoffel: Einige Eigenschaften der Infrarotdarstellungen Diplomarbeit, Hamburg (1973)

[16] T. W. B. Kibble:

Coherent Soft-Photon States and Infrared Divergences.

I. Classical Currents

J. Math. Phys. 9, 315 (1968) 
[17] T. W. B. Kibble:

Coherent Soft-Photon States and Infrared Divergences. III. Asymptotic States and Reduction Formulas

Phys. Rev. 174, 1882 (1968)

[18] S. L. Adler: Consistency Conditions on the Strong Interactions Implied by a Partially Conserved Axial-Vector Current

Phys. Rev. 137B, 1022 (1965)

Consistency Conditions on the Strong Interactions Implied by a Partially Conserved Axial-Vector Current. II.

Phys. Rev. 139B, 1638 (1965)

[19] H. Joos, E. Weimar:

On the Covariant Description of Spontaneously Broken Symmetry in General Field Theory

To be published in N. Cimento

[20] H. Araki, R. Haag:

Collision Cross Sections in Terms of Local Observables:

Commu 2. math. Phys. 4, 77 (1967). 